\title{
Deciphering microRNA targets in pancreatic cancer using miRComb R package
}

\author{
Maria Vila-Casadesús ${ }^{1,2}$, Elena Vila-Navarro ${ }^{1}$, Giulia Raimondi ${ }^{3}$, Cristina Fillat $^{3}$, \\ Antoni Castells ${ }^{1}$, Juan José Lozano ${ }^{1,2}$ and Meritxell Gironella ${ }^{1}$ \\ ${ }^{1}$ Gastrointestinal \& Pancreatic Oncology Group, Centro de Investigación Biomédica en Red de Enfermedades Hepáticas y \\ Digestivas (CIBEREHD), Hospital Clínic of Barcelona, Institut d'Investigacions Biomèdiques August Pi i Sunyer (IDIBAPS), \\ Barcelona, Catalonia, Spain \\ ${ }^{2}$ Bioinformatics Platform, CIBEREHD, Barcelona, Catalonia, Spain \\ ${ }^{3}$ Gene Therapy and Cancer, Institut d'Investigacions Biomèdiques August Pi i Sunyer (IDIBAPS), Centro de Investigación \\ Biomédica en Red de Enfermedades Raras (CIBERER), Universitat de Barcelona, Barcelona, Catalonia, Spain
}

Correspondence to: Meritxell Gironella, email: meritxell.gironella@ciberehd.org

Keywords: microRNA; pancreatic cancer; target prediction; gene expression; CRISPR-Cas9

Received: August 09, $2017 \quad$ Accepted: January 02, $2018 \quad$ Published: January 08, 2018

Copyright: Vila-Casadesús et al. This is an open-access article distributed under the terms of the Creative Commons Attribution License 3.0 (CC BY 3.0), which permits unrestricted use, distribution, and reproduction in any medium, provided the original author and source are credited.

\section{ABSTRACT}

MiRNAs are small non-coding RNAs that post-transcriptionally regulate gene expression. They play important roles in cancer but little is known about the specific functions that each miRNA exerts in each type of cancer. More knowledge about their specific targets is needed to better understand the complexity of molecular networks taking part in cancer. In this study we report the miRNA-mRNA interactome occurring in pancreatic cancer by using a bioinformatic approach called miRComb, which combines tissue expression data with miRNA-target prediction databases (TargetScan, miRSVR and miRDB). MiRNome and transcriptome of 12 human pancreatic tissues (9 pancreatic ductal adenocarcinomas and 3 controls) were analyzed by next-generation sequencing and microarray, respectively. Analysis confirmed differential expression of both miRNAs and mRNAs in cancerous tissue versus control, and unveiled 17401 relevant miRNA-mRNA interactions likely to occur in pancreatic cancer. They were sorted according to the degree of negative correlation between miRNA and mRNA expression. Results highlighted the importance of miR-148a and miR-21 interactions among others. Two components of the Notch signaling pathway, ADAM17 and EP300, were confirmed as miR-148a targets in MiaPaca-2 pancreatic cancer cells overexpressing miR-148a. Moreover, a CRISPR-Cas9 cellular model was generated to knock-out the expression of miR-21 in PANC-1 cells. As expected, the expression of two miRComb miR-21 predicted targets, PDCD4 and BTG2, was significantly upregulated in these cells in comparison to control PANC-1.

\section{INTRODUCTION}

Pancreatic ductal adenocarcinoma (PDAC) is the fourth leading cause of cancer death in occidental countries and has the worst prognosis of all major malignancies with just a $6 \%$ five-year survival rate [1]. By the time of diagnosis, most patients present with locally advanced or metastatic disease that precludes curative resection and have a mean survival of less than 1 year $[2,3]$. This fatal scenario is due, in part, to the high aggressiveness of the tumour and the lack of effective treatments. In order to overcome this dire problem, new and more efficient therapeutic targets are urgently needed. To achieve this goal is highly necessary to increase the knowledge about 
the molecular mechanisms involved in pancreatic cancer and to elucidate intracellular network connections which play indispensable roles for cancer progression.

MicroRNAs (miRNAs) are small endogenous noncoding RNAs of 18-25 nucleotides that negatively regulate gene expression at the posttranscriptional level by either repressing mRNA translation or targeting mRNAs for degradation. One miRNA can modulate up to hundreds of genes and one gene may be regulated by more than one miRNA [4]. MiRNAs are estimated to modulate the translation of more than $60 \%$ of protein-coding genes and are involved in regulating a wide range of biological processes such as cellular proliferation, differentiation, apoptosis and development [5, 6]. Their dysregulation plays an essential role in the development and progression of cancer and they can act as tumour suppressors or oncogenes depending on the target that they are regulating in a specific situation [7].

Aberrant expression of miRNAs has been widely reported in human cancers including $\operatorname{PDAC}[8,9]$. However, the functional meaning of each deregulated miRNA in the context of PDAC is still largely unknown. In order to help with the functional understanding of aberrant miRNomes, we developed an $\mathrm{R}$ package called miRComb [10]. This software is able to combine miRNA and mRNA expression data with hybridization information, in order to find potential miRNA-mRNA targets that are likely to occur in a specific context. In this study, we applied miRComb to combine miRNome and transcriptome expression data from human pancreatic cancer tumor specimens, in order to uncover the miRNAmRNA interactome that is taking place in pancreatic tumorigenesis. The results obtained here will serve to better understand pancreatic tumorigenesis and will help to highlight those miRNA-mRNA interactions that may be playing an important role in this context.

\section{RESULTS}

\section{Data exploration}

The dataset consists on 3 controls (healthy pancreatic tissue samples) and 9 cases (PDAC tissue samples) with paired miRNA-mRNA data, including the expression of 1733 miRNAs and 18570 mRNAs. Supplementary Table 1 shows clinical information related to these patients. Figure 1A shows Principal Components Analysis of the dataset. We can see that PDAC samples are clearly different from healthy ones depending either on miRNA or on mRNA profiling.

\section{Top differentially expressed miRNAs or mRNAs}

Figure 1B shows the most differentially expressed miRNAs and mRNAs between PDAC and healthy tissue. There are 201 significantly upregulated and 342 significantly downregulated miRNAs in our pancreatic cancer set. They represent $31.1 \%$ of the total expressed miRNAs. 30 of the these upregulated miRNAs were validated by RT-qPCR in two larger cohorts of pancreatic cancer patients in our previous article [8]. There also are 1613 significantly upregulated and 2030 significantly downregulated mRNAs between PDAC and healthy tissues, representing $19.6 \%$ of the total expressed mRNAs. Figure $1 \mathrm{C}$ shows their respective volcano plots colouring the miRNAs and mRNAs according to their fold-change (FC). The miRNAs and mRNAs that were selected for further exploration were those with FDR $<0.05$ regardless of their FC (highlighted in yellow, orange and red).

\section{Intersection with miRNA target prediction databases}

We then selected the 543 and 3643 significantly deregulated miRNAs and mRNAs, respectively, and computed all possible correlations. Multiple testing corrections with FDR were applied. Among 1978149 possible miRNA-mRNA combinations, there were 959775 that correlated negatively and, among them, we found 443100 miRNA-mRNA pairs where this correlation had FDR $<0.05$. This number represented $22.4 \%$ of the total miRNA-mRNA possible combinations. Furthermore, we used the information given by 3 miRNA target prediction databases (TargetScan, miRDB, miRSVR) to intersect with the above mentioned correlations.

If we only took into account the 3 miRNA target prediction databases, we would have found a total number of 76878 potential miRNA targets present in at least one of them, and using the interaction calculated by miRComb we reduced this number nearly five times, as we found 17401 miRNA-mRNA pairs that were also negatively correlated in our expression data. That means that only $22.6 \%$ of the miRNA-mRNA interactions appearing in these databases were found as negatively correlated in our dataset. Figure 2A shows the number of negatively correlated miRNA-mRNA pairs, the number of predicted miRNA-mRNA pairs, and the pairs that fulfill both conditions. Figure $2 \mathrm{~B}$ shows how many miRNA-mRNA interactions are predicted by each database among the 17401. We can see that miRSVR provided more of the miRComb predicted miRNA-mRNA pairs than the other databases (10767 in total, while TargetScan and miRDB predicted 2986 and 6897 respectively), probably due to the fact that this database has globally more miRNA-mRNA interactions described than the others.

Only 794 of the negatively correlated miRNAmRNA pairs were simultaneously present in the 3 databases, confirming the little overlap that exists between them. That number corresponds to a $0.88 \%$ of the total miRNA-mRNA possible combinations existing from the tissue expression analysis. That means that this step considerably reduces the number of miRNA target 
interactions that are likely to occur in human pancreatic oncogenesis. Moreover, due to the fact that they have been predicted in the three databases, these interactions could be prioritized ahead of the others in terms of confidence.
Figure 3A shows the network of all these 794 highconfident interactions. MiRNA-mRNA interactions are divided into downregulated miRNAs and their upregulated target mRNAs (left), and upregulated miRNA with

\section{A}

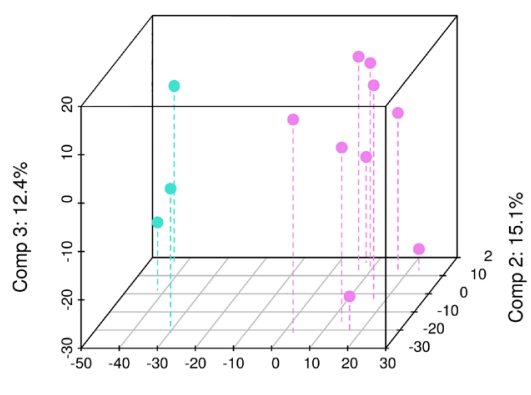

Comp 1: $32.8 \%$

B

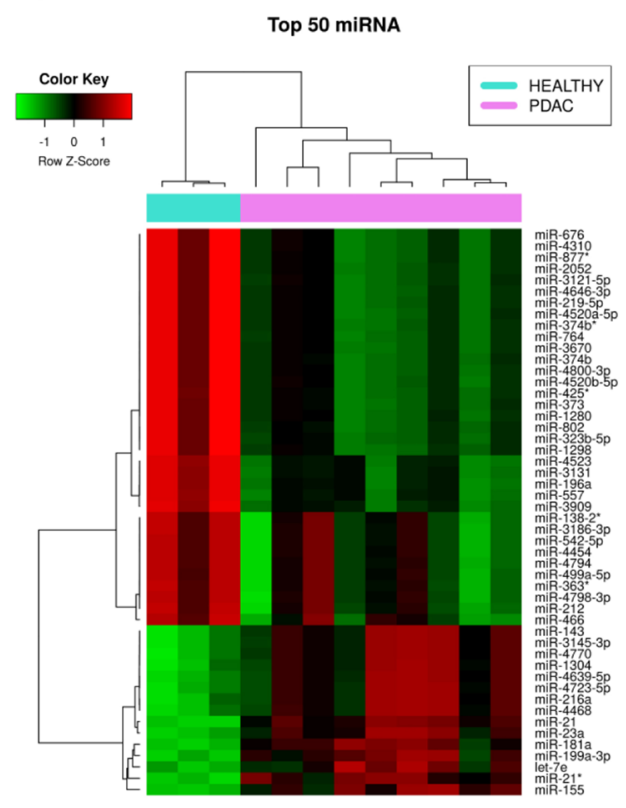

C

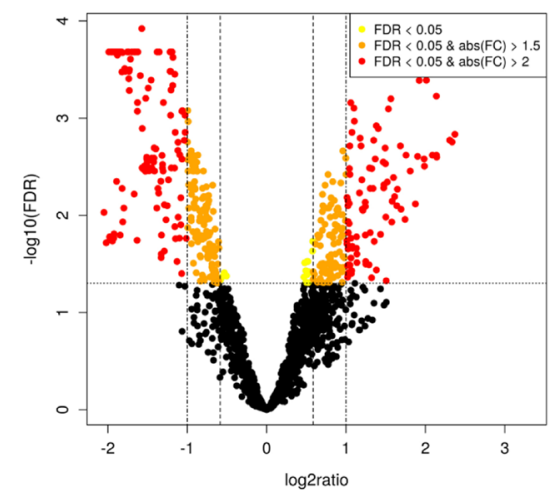

- HEALTHY - PDAC

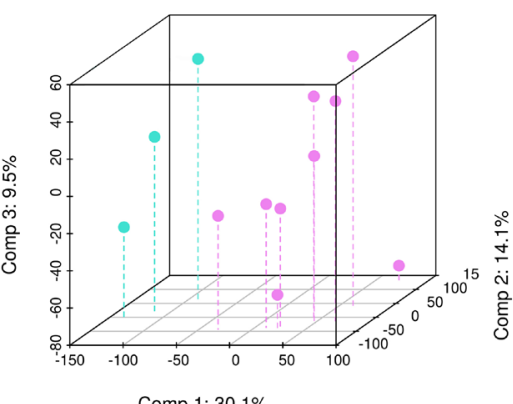

Comp 1: $30.1 \%$
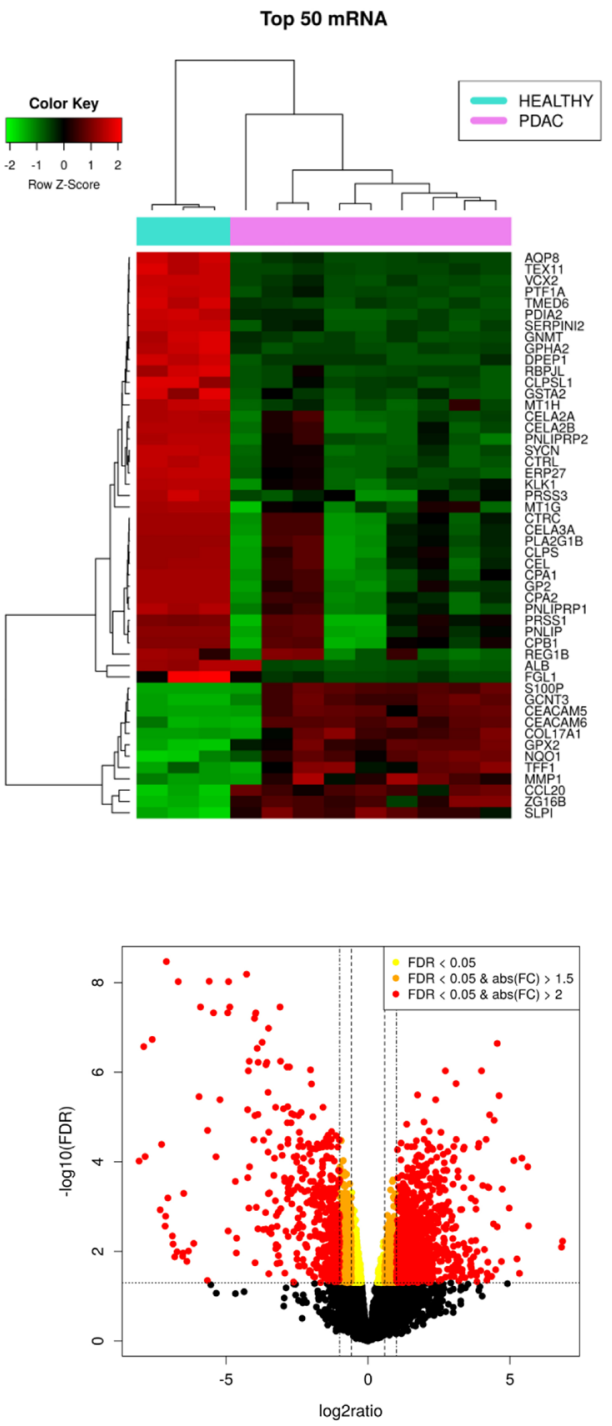

Figure 1: Exploratory analysis of paired miRNA and mRNA expression in pancreatic cancer samples. (A) 3d-Principal Components Analysis plots, based on correlation matrix, for miRNA (left) and mRNA (right) expression in Healthy ( $\mathrm{n}=3$ ) amd PDAC ( $\mathrm{n}=9$ ) tissue samples. (B) Heatmaps of the top 50 most differentially expressed miRNAs and mRNAs, respectively, sorted by absolute FC (all of them having FDR $<0.05$ ). (C) Volcano plot of the miRNAs (left) and mRNAs (right) highlighting in yellow those with FDR $<0.05$, orange $\mathrm{FDR}<0.05$ and absolute $\mathrm{FC}>1.5$, and red FDR $<0.05$ and absolute $\mathrm{FC}>2$. 
downregulated target mRNAs (right). Interestingly, we can see that miRNAs from the same family share most of their target mRNAs, which is why they are represented close to each other. For example, miR-148a and miR-148b are members of the miR-148 family and appear close to each other on the left part of the network (Figure 3A and $3 \mathrm{~B})$. That means they share most of their targets, as it can be observed. Similarly, miR-15a and miR-497 share most of their targets and they belong to the same miR-15 family (Figure $3 \mathrm{~B}$ ). We can also observe, in the right part of Figure 3A, that members of the let-7 family (let-7a/c/d/ e/f and miR-98) are clustered together according to their miRComb predicted miRNA-mRNA interactions (Figure $3 C)$. In the same way, miR-181a and miR-181b, members of the same miR-181 family, appear together sharing most of their targets. The same occurs for miR-93 and miR$106 \mathrm{~b}$ that belong to the same family, for miR-320a and miR-320b and for miR-19a and miR-19b (right part Figure $3 \mathrm{~A})$ that are clustered together, respectively, according to their miRComb targets.

\section{MiRComb results in the pancreatic cancer set}

Supplementary Table 2 shows all 17401 significant miRNA-mRNA interactions found by MiRComb in our pancreatic cancer set of samples. The most significant
miRNA-mRNA interactions are detailed below. Table 1 shows the top 50 miRNA-mRNA pairs with most significant negative correlations obtained from miRComb, that appear simultaneously in the three mentioned databases. These would be the miRNA-mRNA interactions that are more likely to occur in a pancreatic cancer context. Figure 4 shows miRNA-mRNA expression correlation for the first 12 most significant miRNA-mRNA pairs of that table. These miRNA-mRNA interactions are miR106b-LRRC55, miR-21-PDCD4, miR-148a-YWHAB, miR-93-FAM129A, miR-330-5p-GPI, miR-330-5pBHLHE40, miR-93-LRIG1, miR-23a-LRIG1, miR-148aARF4, miR-106b-FAM129A, miR-148a-ACVR1, miR148a-CTTNBP2NL. PDCD4, GPI, and BHLHE4 are proteins with a described role in pancreatic cancer and, consequently, information about factors that can modulate their expression is important. Concerning the other targets that have not been yet related to pancreatic cancer, this information provides knowledge about new potential pathways playing a role in PDAC. Interestingly, among the miRNAs participating in the 50 most significant miRNA-mRNA interactions we can find: miR-106b, miR93, miR-148a, miR-330-5p that could be interacting with more than 4 different targets at the same time.

Table 2 shows the top 10 miRNAs according to its number of targets predicted by miRComb, appearing
A

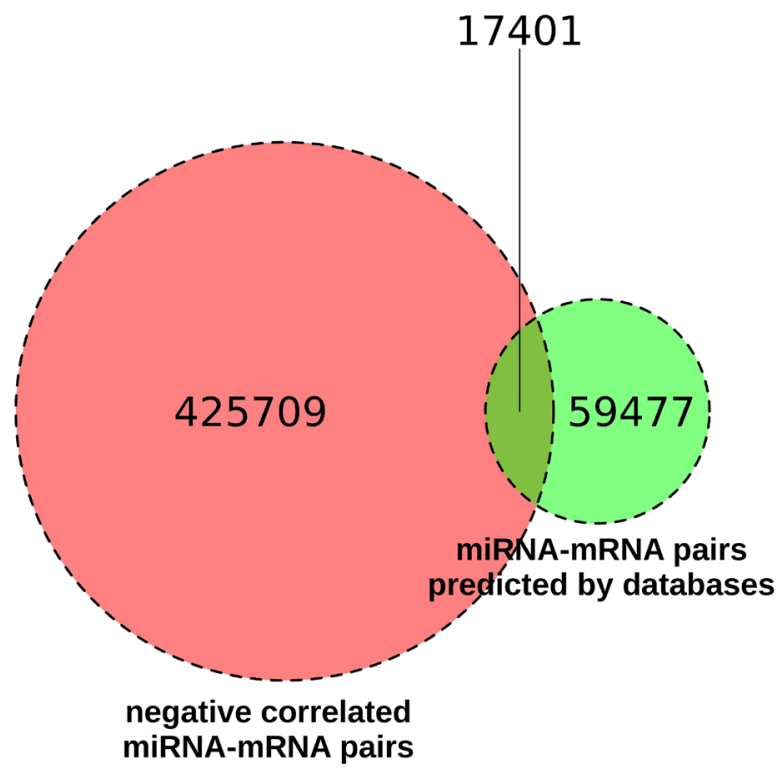

B

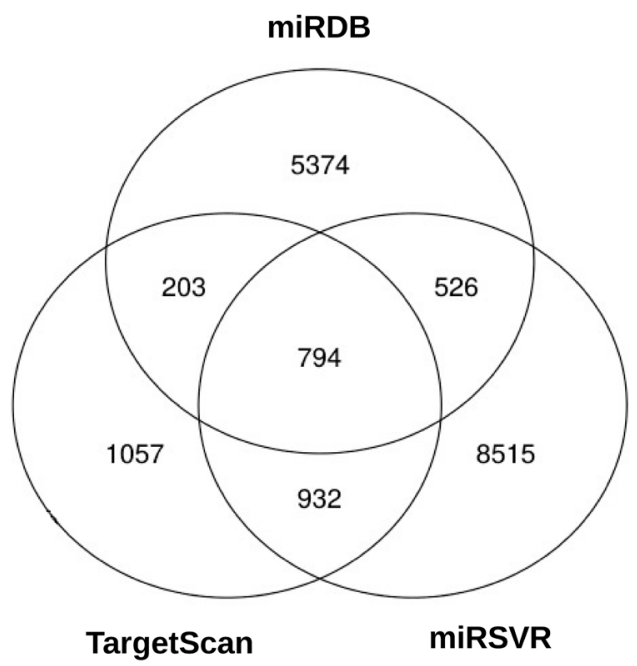

Figure 2: Venn diagrams about the number of miRNA-mRNA interactions predicted by miRComb in the pancreatic cancer context. (A) Negatively correlated miRNA-mRNA pairs (FDR $<0.05$, left), pairs predicted by at least one database (TargetScan, miRDB or miRSVR, right), and miRNA-mRNA pairs that fulfill both conditions. (B) Venn diagram showing the overlap between databases among the 17401 miRNA-mRNA pairs that are negatively correlated (FDR $<0.05)$ and predicted in at least one database (TargetScan, miRDB or miRSVR). 
in at least 1 of the used databases. We have added a column showing the number of potential targets initially predicted by the pre-existing target prediction databases, indicating the reduction of the number of miRNA-mRNA interactions provided by miRComb. Each miRNA show different degrees of reduction, being $77 \%$ the global average percentage of reduction. It is worth noting that these 10 miRNAs together (miR-374b, miR-148a, miR181a, miR-373, miR-320a, miR-448, miR-93, miR-106b, miR-217, miR-539) could potentially be regulating $41 \%$ of the mRNAs significantly altered in PDAC. Conversely, Table 3 shows the top 10 mRNAs with more miRNAs targeting them.

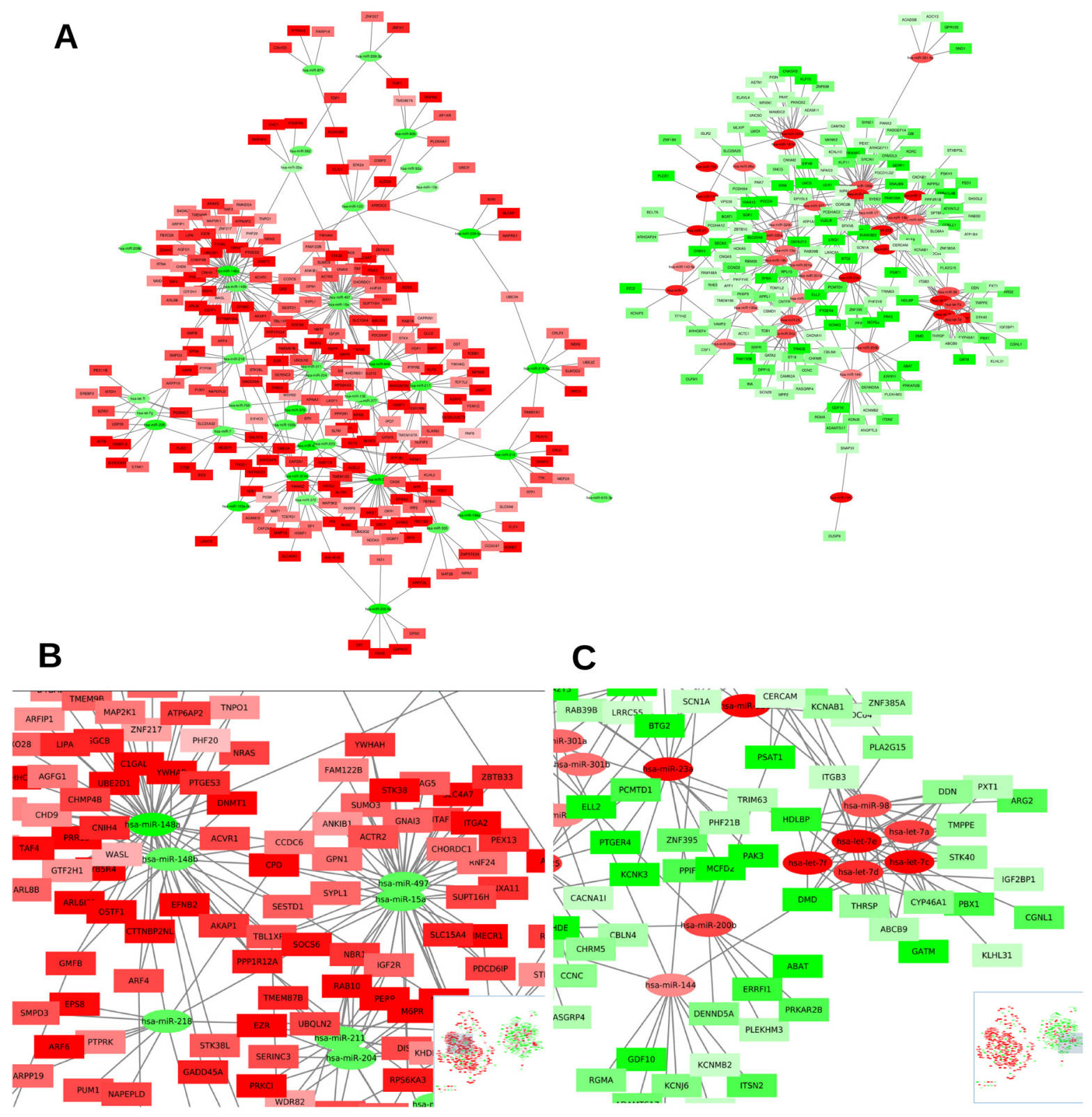

Figure 3: Network of high-confident occurring miRNA-mRNA interactions in pancreatic cancer. (A) Network of the 794 high-confident miRNA-mRNA interactions occurring in our pancreatic cancer dataset (negatively correlated -FDR $<0.05$ - and predicted simultaneously in the three used databases: TargetScan, miRSVR and miRDB). Circles represent miRNAs and squares mRNAs, red fill means upregulated miRNA or mRNA, while green fill means downregulated miRNA or mRNA (color intensity is proportional to the FC), lines indicate miRNA-mRNA miRComb interactions. (B) Zoom of a left A plot region highlighting the mRNA interactions found for miR148 family. (C) Zoom of a right A plot region highlighting the mRNA interactions found for let-7 family. 
Table 1: Top 50 miRNA-mRNA interactions predicted by miRComb

\begin{tabular}{|c|c|c|c|c|c|c|}
\hline miRNA & mRNA & cor & FDR & FC.miRNA & FC.mRNA & dat.sum \\
\hline miR-106b & LRRC55 & $-0,97$ & 0,009 & 2,07 & $-1,23$ & 3 \\
\hline miR-21 & PDCD4 & $-0,93$ & 0,010 & 9,91 & $-7,90$ & 3 \\
\hline $\operatorname{miR}-148 \mathrm{a}$ & YWHAB & $-0,93$ & 0,010 & $-2,98$ & 3,11 & 3 \\
\hline miR-93 & FAM129A & $-0,92$ & 0,010 & 2,60 & $-11,48$ & 3 \\
\hline $\operatorname{miR}-330-5 p$ & GPI & $-0,91$ & 0,011 & $-3,64$ & 3,38 & 3 \\
\hline miR-330-5p & BHLHE40 & $-0,91$ & 0,011 & $-3,64$ & 7,97 & 3 \\
\hline miR-93 & LRIG1 & $-0,91$ & 0,011 & 2,60 & $-4,13$ & 3 \\
\hline miR-23a & LRIG1 & $-0,91$ & 0,011 & 4,40 & $-4,13$ & 3 \\
\hline miR-148a & ARF4 & $-0,91$ & 0,011 & $-2,98$ & 2,11 & 3 \\
\hline miR-106b & FAM129A & $-0,90$ & 0,011 & 2,07 & $-11,48$ & 3 \\
\hline $\operatorname{miR}-148 \mathrm{a}$ & ACVR1 & $-0,90$ & 0,012 & $-2,98$ & 2,11 & 3 \\
\hline miR-148a & CTTNBP2NL & $-0,90$ & 0,012 & $-2,98$ & 2,76 & 3 \\
\hline miR-107 & PDK4 & $-0,90$ & 0,012 & 2,08 & $-12,85$ & 3 \\
\hline $\operatorname{miR}-106 b$ & LMO3 & $-0,89$ & 0,012 & 2,07 & $-4,07$ & 3 \\
\hline $\operatorname{miR}-148 \mathrm{a}$ & C1GALT1 & $-0,89$ & 0,012 & $-2,98$ & 6,38 & 3 \\
\hline $\operatorname{miR}-330-5 p$ & CAPN12 & $-0,89$ & 0,012 & $-3,64$ & 3,99 & 3 \\
\hline miR-148a & TBL1XR1 & $-0,89$ & 0,013 & $-2,98$ & 2,06 & 3 \\
\hline miR-320b & KIAA1324 & $-0,89$ & 0,013 & 1,66 & $-12,22$ & 3 \\
\hline miR-320a & LMO3 & $-0,88$ & 0,013 & 2,14 & $-4,07$ & 3 \\
\hline miR-93 & SCN1A & $-0,88$ & 0,014 & 2,60 & $-1,25$ & 3 \\
\hline miR-148a & CNIH4 & $-0,87$ & 0,014 & $-2,98$ & 2,46 & 3 \\
\hline $\operatorname{miR}-148 \mathrm{a}$ & DNMT1 & $-0,87$ & 0,014 & $-2,98$ & 3,09 & 3 \\
\hline miR-320b & RPL15 & $-0,87$ & 0,014 & 1,66 & $-2,09$ & 3 \\
\hline miR-193b & TNFRSF 21 & $-0,87$ & 0,014 & $-2,05$ & 8,10 & 3 \\
\hline $\operatorname{miR}-148 \mathrm{a}$ & UBE2D1 & $-0,87$ & 0,014 & $-2,98$ & 3,74 & 3 \\
\hline miR-181a & LMO3 & $-0,87$ & 0,014 & 5,17 & $-4,07$ & 3 \\
\hline miR-193b & YWHAZ & $-0,87$ & 0,014 & $-2,05$ & 2,59 & 3 \\
\hline miR-424 & LRIG1 & $-0,86$ & 0,014 & 1,82 & $-4,13$ & 3 \\
\hline miR-106b & PDCD1LG2 & $-0,86$ & 0,014 & 2,07 & $-1,30$ & 3 \\
\hline miR-130a & LRIG1 & $-0,86$ & 0,015 & 1,74 & $-4,13$ & 3 \\
\hline miR-497 & ITGA2 & $-0,86$ & 0,015 & $-1,94$ & 23,44 & 3 \\
\hline miR-15a & ITGA2 & $-0,86$ & 0,015 & $-1,96$ & 23,44 & 3 \\
\hline miR-34a & VAMP2 & $-0,86$ & 0,015 & 2,05 & $-1,50$ & 3 \\
\hline miR-155 & SCN1A & $-0,86$ & 0,015 & 4,03 & $-1,25$ & 3 \\
\hline miR-299-3p & TOP1 & $-0,86$ & 0,015 & $-1,87$ & 2,35 & 3 \\
\hline miR-367 & TOB1 & $-0,86$ & 0,015 & 1,61 & $-1,60$ & 3 \\
\hline miR-330-5p & ARPC5L & $-0,86$ & 0,015 & $-3,64$ & 3,17 & 3 \\
\hline
\end{tabular}




\begin{tabular}{lcccccc}
\hline miRNA & mRNA & cor & FDR & FC.miRNA & FC.mRNA & dat.sum \\
\hline miR-19b & RBM20 & $-0,86$ & 0,015 & 2,00 & $-1,80$ & 3 \\
miR-34a & INA & $-0,86$ & 0,015 & 2,05 & $-1,72$ & 3 \\
miR-148a & CPD & $-0,86$ & 0,015 & $-2,98$ & 3,44 & 3 \\
miR-148a & GMFB & $-0,86$ & 0,015 & $-2,98$ & 2,37 & 3 \\
miR-374b & NMT1 & $-0,86$ & 0,015 & $-3,79$ & 1,71 & 3 \\
miR-373 & RAB11A & $-0,86$ & 0,015 & $-3,76$ & 3,29 & 3 \\
miR-374b & TCERG1 & $-0,85$ & 0,015 & $-3,79$ & 1,59 & 3 \\
miR-373 & CAPZA1 & $-0,85$ & 0,015 & $-3,76$ & 2,30 & 3 \\
miR-373 & PFKP & $-0,85$ & 0,015 & $-3,76$ & 14,95 & 3 \\
miR-144 & ANGPTL3 & $-0,85$ & 0,015 & 1,63 & $-1,27$ & 3 \\
miR-19b & SLC25A6 & $-0,85$ & 0,015 & 2,00 & $-3,36$ & 3 \\
miR-93 & PDCD1LG2 & $-0,85$ & 0,015 & 2,60 & $-1,30$ & 3 \\
miR-148a & EFNB2 & $-0,85$ & 0,015 & $-2,98$ & 3,80 & 3 \\
\hline
\end{tabular}

MiRNA-mRNA interactions are sorted by FDR and are predicted simultaneously in the three used databases (TargetScan, miRSVR and miRDB; dat.sum=3).

As an overview, Figure 5A shows the number of mRNA targets per each miRNA and the cumulative number of mRNAs that are being regulated by the previous miRNAs. Interestingly, $50 \%$ of the significantly deregulated mRNAs are regulated by the top 17 miRNAs, and almost no mRNAs are added by the last ones. Moreover, Figure 5B shows the number of mRNAs targeted by 0,1 or more miRNAs. It is important to point out that 1149 mRNAs (representing $41.7 \%$ of the significantly deregulated ones in PDAC samples) are targeted by more than 5 miRNAs. Furthermore, both figures show that $75 \%$ of the significantly deregulated mRNAs are targeted by at least one miRNA.

It is interesting to highlight that miR-148a also appears on the top list from Table 2, emphasizing its importance in pancreatic carcinogenesis. Target enrichment analysis of these miR-148a targets by KEGG revealed significant enrichment only in the Notch Signaling Pathway $($ FDR $<0.02)$. Consistently, target enrichment analysis with other methods as GO-Biological Processes or Reactome also showed significant enrichment in this Notch pathway among others. Supplementary Table 3 shows all the results obtained from the three different target enrichment analysis performed. Figure 6 shows as 7 key members (NUMB, DTX4, DTX3L, PSEN1, APH1A, ADAM17 and EP300) of that pathway are predicted as miRComb miR-148a targets in our pancreatic cancer samples.

\section{Assessment of miR-148a targets from Notch pathway in a pancreatic cancer cellular model}

In order to check those proposed miR-148a targets in the context of pancreatic cancer we took advantage of the pancreatic cancer cellular model (MiaPaCa-2) stably overexpressing miR-148a, that we have previously generated [11]. We measured the expression of key members of the Notch Signaling Pathway (NUMB, DTX4, DTX3L, PSEN1, APH1A, ADAM17 and EP300) in the MiaPaCa-2-miR-148a by qRT-PCR, and compared it with the basal levels of the control pancreatic cancer cell line MiaPaCa-2, expressing very low levels of miR-148a (Figure 7A). Among the seven targets analyzed, ADAM17 and EP300, showed significantly decreased expression in the presence of high levels of miR-148a compared to the low miR-148a levels expressed by the control cell line (Figure 7B and 7C). These results show that the expression of Notch Signaling components ADAM17 and EP300 is, at least in part, regulated by miR-148a in a pancreatic cancer context.

\section{Assessment of miR-21 targets in a pancreatic cancer cellular model}

To go one step further, we focused on miR-21 because it is one of the most up-regulated miRNAs in PDAC as we can see in Table 1 with a $\mathrm{FC}=9.91$. This wide range of expression makes it a good candidate to test some of its mRNA targets. In Table 4 is shown the list of top miRComb predicted targets for miR-21 (present in more than 1 database). In order to experimentally evaluate some of these miR-21 predicted targets in the context of pancreatic cancer, we selected PDCD4 and BTG2 from that list for being also highly down-regulated in PDAC ( $\mathrm{FC}=-7.88$ and $\mathrm{FC}=-5.53$, respectively). We generated a pancreatic cancer cellular model (PANC-1) lacking miR- 


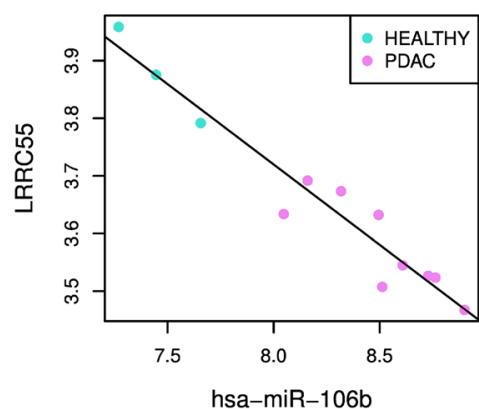

Pearson Cor.: -0.915; adj.pval: 0.01

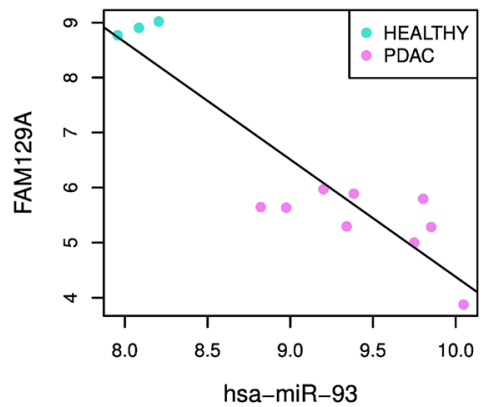

Pearson Cor.: -0.906; adj.pval: 0.011

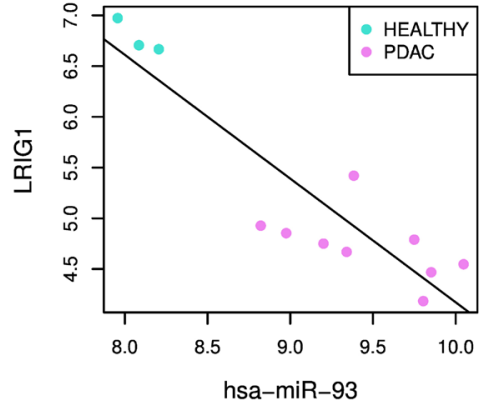

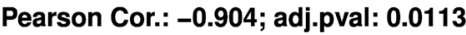

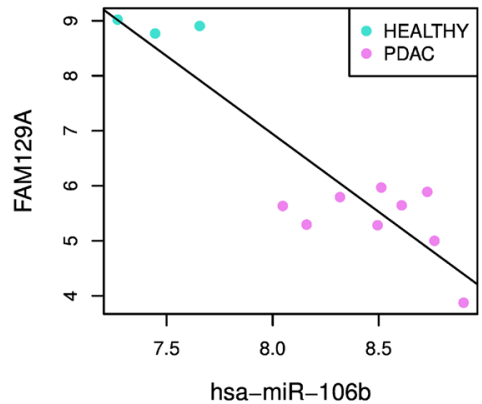

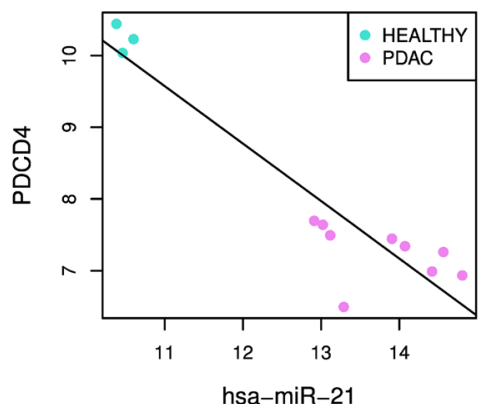

Pearson Cor.: -0.909; adj.pval: 0.0105

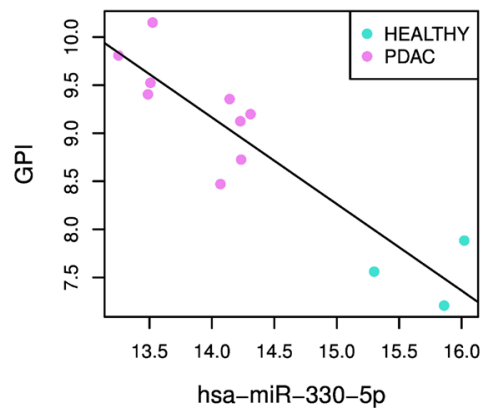

Pearson Cor.: -0.906; adj.pval: 0.011

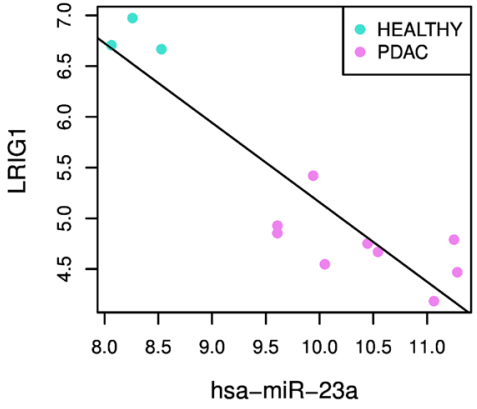

Pearson Cor.: $\mathbf{- 0 . 9}$; adj.pval: $\mathbf{0 . 0 1 1 7}$

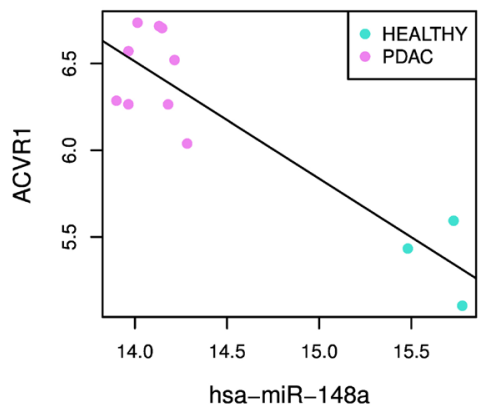

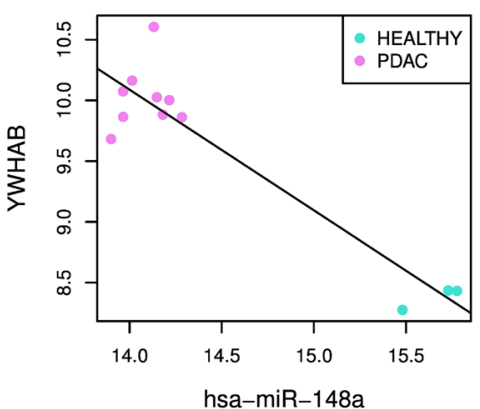

Pearson Cor.: -0.909; adj.pval: 0.0106

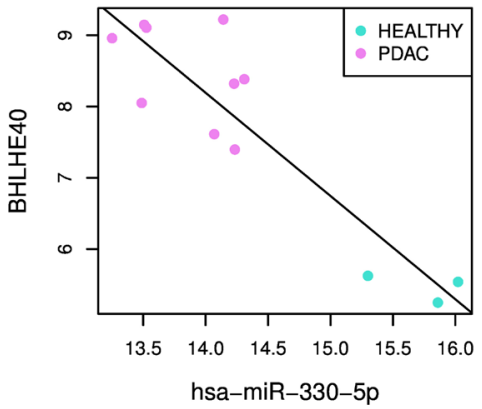

Pearson Cor.: -0.905; adj.pval: 0.0111

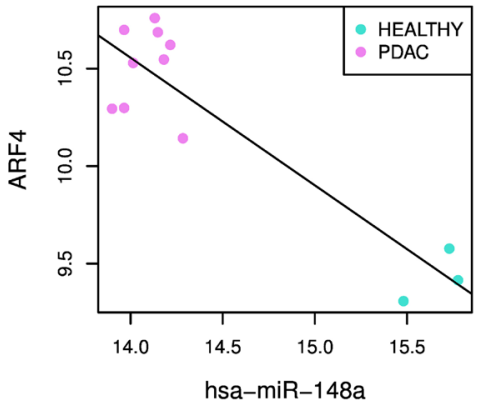

Pearson Cor.: $\mathbf{- 0 . 8 9 9}$; adj.pval: $\mathbf{0 . 0 1 1 9}$

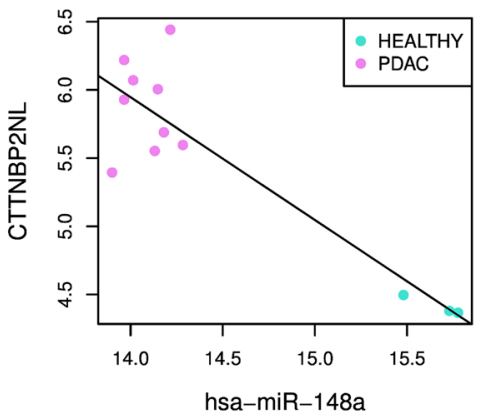

Figure 4: Plot of the top 12 miRNA-mRNA miRComb interactions occurring in pancreatic cancer. All miRNA-mRNA pairs are negatively correlated, sorted by correlation FDR, FDR $<0.05$ and predicted simultaneously in the three used databases: TargetScan, miRSVR and miRDB. 
Table 2: Top 10 miRNAs by number of targets

\begin{tabular}{|c|c|c|c|c|}
\hline miRNA & \#tgts & Orig & Cum \% & Names of mRNA targets (Top 20) \\
\hline$m i R-374 b$ & 381 & $(866,56 \%)$ & $10,46 \%$ & $\begin{array}{c}\text { PMEPA1, CD58, TMSB10, CCL20, CTSB, HSPH1, } \\
\text { DNMT1, DIS3, ELF1, UBAC2, FAT1, CCDC47, } \\
\text { PTPN12, COPB1, FAM122B, IL8, CTTNBP2NL, } \\
\text { FAM96A, H2AFY, ACVR1 }\end{array}$ \\
\hline$m i R-148 a$ & 363 & $(595,39 \%)$ & $16,85 \%$ & $\begin{array}{l}\text { HLA-A, KLF5, CTSB, TNFRSF21, TMSB10, BID, } \\
\text { TMEM123, KCNK1, B2M, PGRMC1, YWHAB, } \\
\text { TAGLN2, ENDOD1, PTPN12, UBE2A, ACSL3, } \\
\text { MYO1D, AMMECR1, PLEKHB2, ACTG1 }\end{array}$ \\
\hline miR-181a & 259 & $(828,69 \%)$ & $23,96 \%$ & $\begin{array}{l}\text { PDCD4, IFRD1, DFFB, EPB41L4B, ANGPT1, LRIG1, } \\
\text { KCNN1, NUCB2, DMGDH, FKBP11, EPB41, TMED6, } \\
\text { LMO3, VCX2, MYO15A, RPL15, SLC25A53, PSAT1, } \\
\text { ITSN2, SPATA20 }\end{array}$ \\
\hline$m i R-373$ & 258 & $(647,60 \%)$ & $26,52 \%$ & $\begin{array}{c}\text { HLA-A, ENDOD1, B2M, PGRMC1, BID, DIS3, } \\
\text { TAGLN2, CCDC47, PTPN12, MDK, PON2, MYO1D, } \\
\text { SKAP2, CTTNBP2NL, FAM96A, IL8, H2AFY, PSMA2, } \\
\text { ACVR1, C1D }\end{array}$ \\
\hline miR-320a & 252 & $(751,66 \%)$ & $31,62 \%$ & $\begin{array}{c}\text { WNT9B, PDCD4, TMED6, PAIP2B, SFTPC, ADRA1B, } \\
\text { MS4A10, HHIPL1, CACNB1, AOX1, IFRD1, SND1, } \\
\text { CECR2, GPHA2, KCNAB1, OSBP2, ERO1LB, } \\
\text { EPB41L4B, LMO3, BACE1 }\end{array}$ \\
\hline$m i R-448$ & 245 & $(608,60 \%)$ & $33,24 \%$ & $\begin{array}{c}\text { ENDOD1, GBP2, LITAF, LIMS1, DNMT1, ELF1, } \\
\text { PTPN12, IL8, FAM96A, VPS13C, SEPT10, SKAP2, } \\
\text { CTTNBP2NL, FAM122B, CALM2, RBM41, PPFIA1, } \\
\text { IVNS1ABP, NEK6, PFKP }\end{array}$ \\
\hline miR-93 & 238 & $(813,71 \%)$ & $36,62 \%$ & $\begin{array}{l}\text { IFRD1, FAM129A, LRIG1, ATXN7L2, MLC1, } \\
\text { EPB41L4B, SH2D5, ANGPT1, ISM2, MS4A10, SYBU, } \\
\text { SCN1A, MYO15A, PCMTD1, FBXO24, SLC46A2, } \\
\text { EPB41, ITSN2, PAIP2B, WNT9B }\end{array}$ \\
\hline miR-106b & 234 & $(766,69 \%)$ & $37,47 \%$ & $\begin{array}{l}\text { LRRC55, FNDC5, ZNF385A, SH2D5, FAM129A, } \\
\text { MYT1, MLC1, LMO3, IFRD1, C17orf67, KPNA7, } \\
\text { APOBEC3H, SLC41A1, TIMM8A, ATOH8, PAIP2B, } \\
\text { ARHGAP18, ERO1LB, PRND, MUM1L1 }\end{array}$ \\
\hline$m i R-217$ & 230 & $(533,57 \%)$ & $39,28 \%$ & $\begin{array}{l}\text { TNFRSF21, CTNNA1, ARPC2, CLINT1, RAB11A, } \\
\text { YWHAH, KLF5, PFKP, MAP4K4, YWHAB, CAP1, } \\
\text { PTTG1IP, RAC1, SPTLC2, ADAM9, PRKCI, ISG20, } \\
\text { TES, DDX60, TMEM87B }\end{array}$ \\
\hline$m i R-539$ & 225 & $(868,74 \%)$ & $41,23 \%$ & $\begin{array}{l}\text { CCDC109B, NQO1, SULF2, KCNK1, MARCKSL1, } \\
\text { ITGA2, PSMB8, ARPC2, DENND2D, HSBP1, } \\
\text { SLC44A1, MRPL50, B2M, ENC1, FAM108C1, MAT2B, } \\
\text { GCC2, HLA-A, DYNLT1, PNP }\end{array}$ \\
\hline
\end{tabular}

Top 10 miRNA with more targets (each miRNA-mRNA pair has FDR $<0.05$ and appears at least 1 times in the following databases: TargetSan, miRSVR, miRDB). MiRNAs in bold are upregulated in PDAC, miRNAs in italics are downregulated in PDAC. \#tgts: Number of target mRNAs; orig.: number of miRNA-mRNA pairs predicted in at least one database (considering positive and negatively correlated miRNA-mRNA pairs) and percentage of these original pairs that are removed after considering only negatively correlated miRNA-mRNA pairs. Cum \%: percentage of deregulated mRNAs that are regulated by the miRNAs, cumulatively. Names of the top 20 mRNA targets are sorted by correlation. 
Table 3: Top 10 mRNAs by number of miRNAs

mRNA \#miRNA tgts

TBL1XR1

CTTNBP2NL

YWHAZ

36

AMMECR1

TNPO1

CCDC6

CPD

CPSF6

G3BP2

PDCD6IP
Names of miRNA (Top 20)

miR-148a, miR-148a ${ }^{*}$, miR-4712-3p, miR-3666, miR-217, miR-4668-5p, miR-4429, miR-15a, miR-497, miR-619, miR-377, miR-5481, miR-211, miR-876-5p, miR- 338-3p, miR-148b, miR-548n, miR-548f, miR-548g, miR-4474-3p

miR-148a, miR-2052, miR-3167, miR-373, miR-374b, miR-448, miR-3305p, miR-4463, miR-196a, miR-302c ${ }^{*}$, miR-567, miR-3168, miR-323-3p, miR-891b, miR-193b, miR-372, miR-377, miR-876-5p, miR-122, miR-136

miR-193b, miR-217, miR-4429, miR-375, miR-339-5p, miR-636, miR122, miR-758, miR-4474-3p, miR-92b, miR-204, miR-876-5p, miR-136, miR-548am, miR-211, miR-802, miR-641, miR-448, miR-7, miR-373

miR-148a, miR-196a, miR-4310, miR-4253, miR-4700- 5p, miR-448, miR-618, miR-4436b-5p, miR-1236, miR- 4428, miR-4668-5p, miR-497, miR-15a, miR-876-5p, miR-148b, miR-548g, miR-548n, miR-4679, miR-

548am, miR-548m

miR-299-3p, miR-154, miR-211, miR-4668-5p, miR- 325, miR-4418, miR-218, miR-548n, miR-208b, miR-148a, miR-4469, miR-15a, miR548f, miR-548g, miR- 497, miR-4504, miR-548m, miR-548h, miR-548am, miR-4775

miR-302c*, miR-567, miR-148a, miR-30a*, miR-4725- 3p, miR-148a*, miR-3666, miR-641, miR-4310, miR-373, miR-802, miR-374b*, miR3685, miR-875-5p, miR-330-5p, miR-557, miR-497, miR-15a, miR-122, miR-211

miR-148a, miR-30a*, miR-635, miR-373, miR-641, miR-196a, miR-448, miR-497, miR-15a, miR-211, miR-377, miR-4255, miR-378e, miR-548f, miR-876-5p, miR-148b, miR-548am, miR-204, miR-338-3p, miR-4477b

miR-148a, miR-4761-3p, miR-4741, miR-497, miR-15a, miR-802, miR377, miR-204, miR-548f, miR- 548am, miR-548n, miR-670, miR-136, miR-4762-3p, miR-548m, miR-448, miR-548h, miR-618, miR-4775, miR2355-3p

miR-148a, miR-374b, miR-802, miR-4253, miR-448, miR-219-5p, miR4463, miR-217, miR-4688, miR-3184, miR-148a*, miR-323-3p, miR485-3p, miR-497, miR- 4668-5p, miR-15a, miR-122, miR-212, miR-335, miR-148b

miR-148a, miR-217, miR-26b*, miR-3144-3p, miR- 323-3p, miR-5481, miR-148b, miR-211, miR-15a, miR-497, miR-876-5p, miR-4477b, miR548g, miR-204, miR-875-5p, miR-3140-5p, miR-4262, miR-4775, let-7i, miR-485-3p

Top 10 mRNA with more miRNAs targeting them (each miRNA-mRNA pair has FDR $<0.05$ and appears at least 1 times in the following databases: TargetScan, miRSVR, miRDB). mRNAs in bold are upregulated in PDAC. Names of the top 20 miRNA targeting the mRNA are sorted by correlation.

21 expression by using CRISPR/Cas9 methodology. After confirming which clones did not express miR-21 (Figure $8 \mathrm{~A})$, we measured the basal expression of PDCD4 and BTG2 in three PANC-1 KO miR-21 clones (c4, c5 and c6), and compared it with the control pancreatic cancer cell line PANC-1 expressing high levels of miR-21. As expected, both, PDC4 and BTG2, showed significantly increased expression in the absence of miR-21 compared to the control miR-21 expressing cell line (Figure 8B, 8C). These results show that the expression of these genes is, at least in part, regulated by miR-21 in a pancreatic cancer context. 

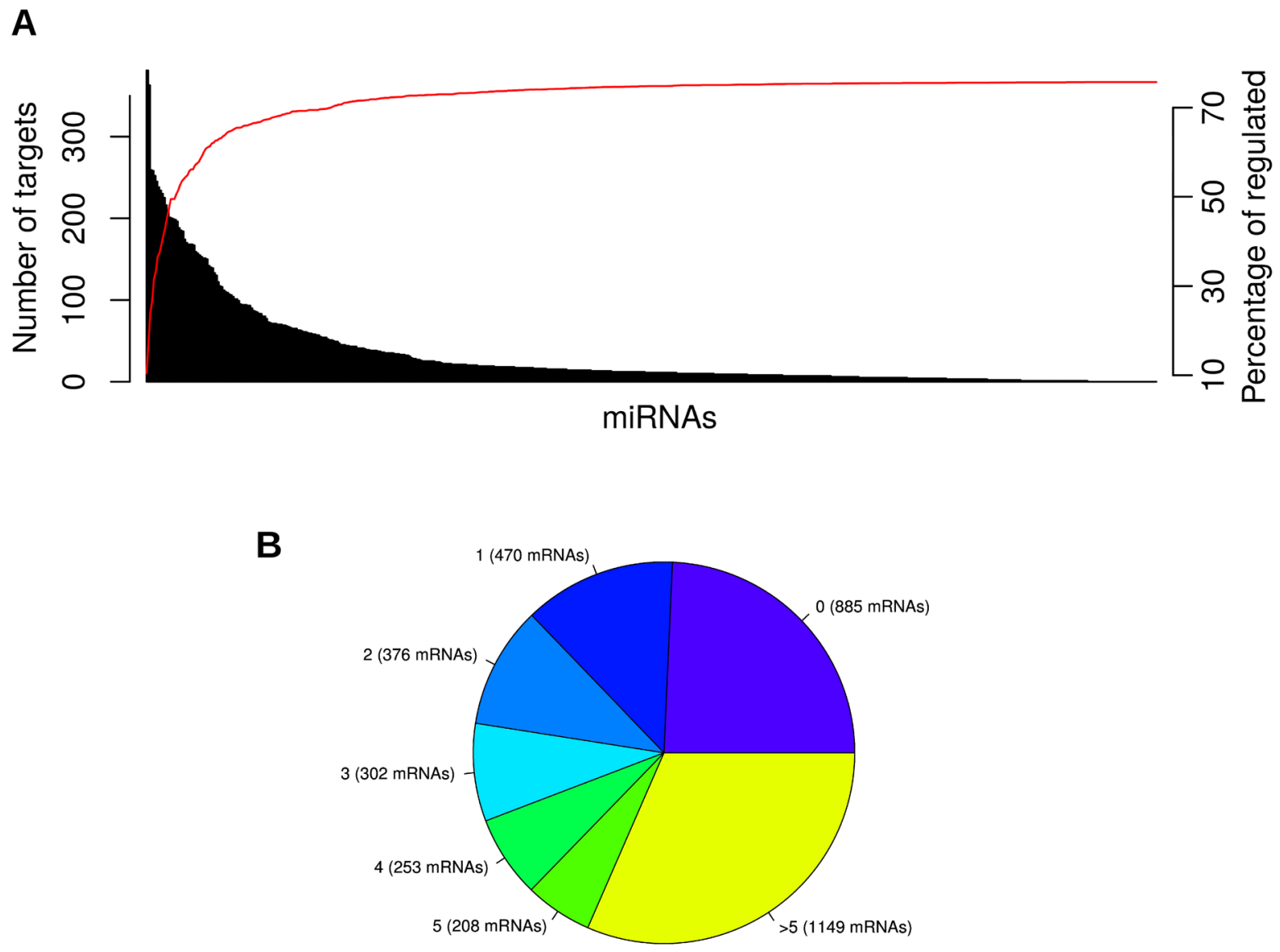

Figure 5: Barplot an piechart summarizing the number of miRComb interactions per miRNA and mRNA. MiRNAmRNA miRComb interactions are those negatively correlated (FDR $<0.05)$ and predicted in at least one database (TargetScan, miRVR or miRDB). (A) Barplot showing the number of mRNA targets per each miRNA (each bar represents a miRNA and they are sorted by number of targets). Red line means the percentage of mRNAs that are cumulatively regulated by the previous miRNAs. (B) Pie chart representing the number of miRNAs that are regulating each mRNA.

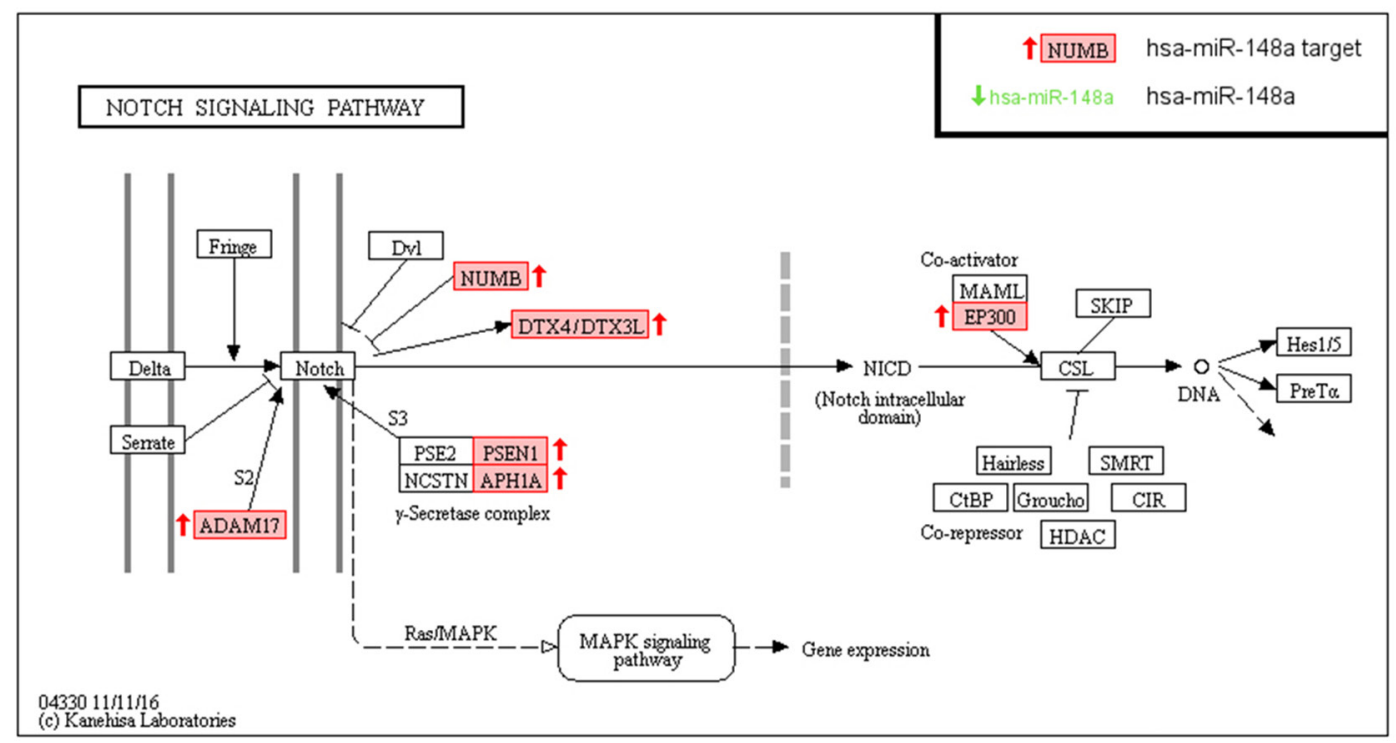

Figure 6: MiR-148a targets involved in Notch signaling pathway from KEGG analysis, in the context of pancreatic cancer. miR-148a miRComb targets (mRNAs that are negatively correlated with miR-148a -FDR $<0.05-$ and predicted in at least one database of TargetScan, miRVR or miRDB) are highlighted in red. 


\section{DISCUSSION}

In this study we have shown that the final miRComb number of miRNA-mRNA interactions in our set of pancreatic cancer samples is 17401 , that corresponds to a $22.6 \%$ of the 76878 potential miRNA targets predicted by at least one of the miRNA target prediction databases used in this study (TargetScan, miRSVR, miRDB). The expression of these 17401 miRNA-mRNA pairs is negatively correlated (FDR $<0.05)$ and predicted in at least one of the above mentioned databases. Although the experimental confirmation of all these interactions have not been done, and therefore there may be some false positives among them, this analysis considerably filters the number of potential miRNA target interactions and help to focus more directly on those that are more likely to occur in pancreatic carcinogenesis.

The little overlap found on the predictions of the three mentioned databases reinforces the idea that is better to use more than one database and take advantage of other sources of information such as miRNA and mRNA expression to filter out the results. Concordantly, previous studies suggest that combinations of miRNA-mRNA databases have less false positives [12]. In our study,

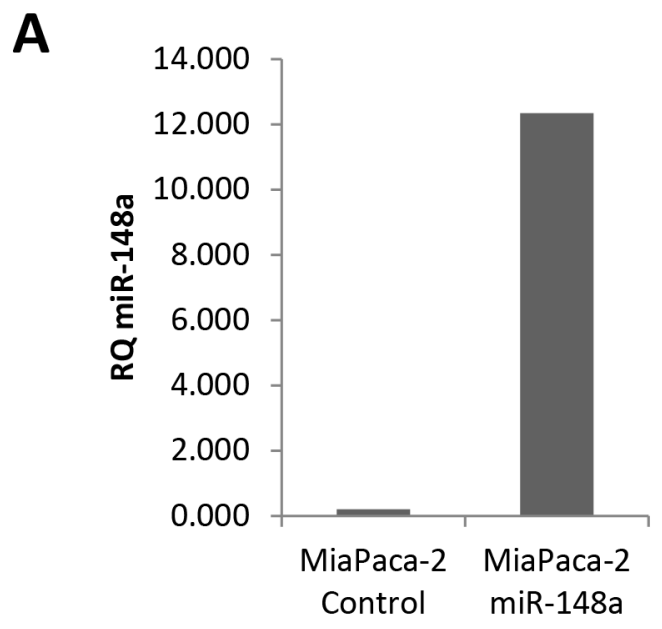

B

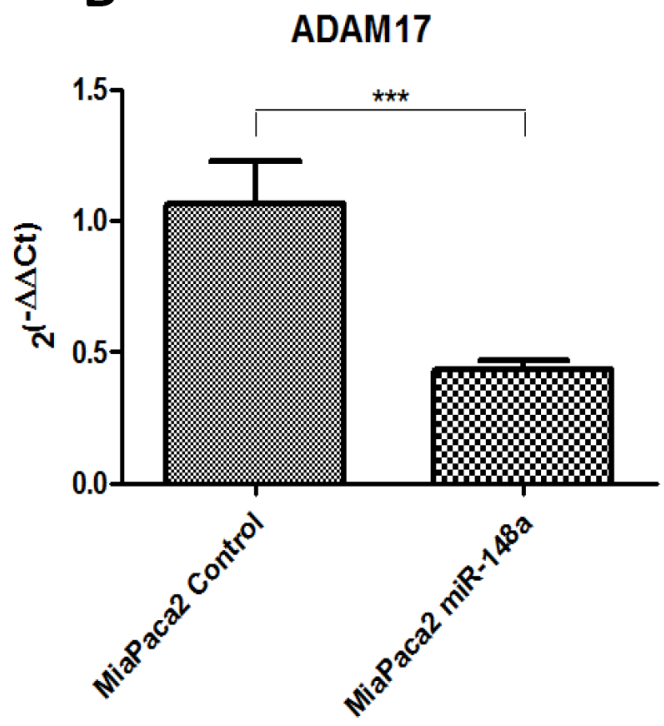

C

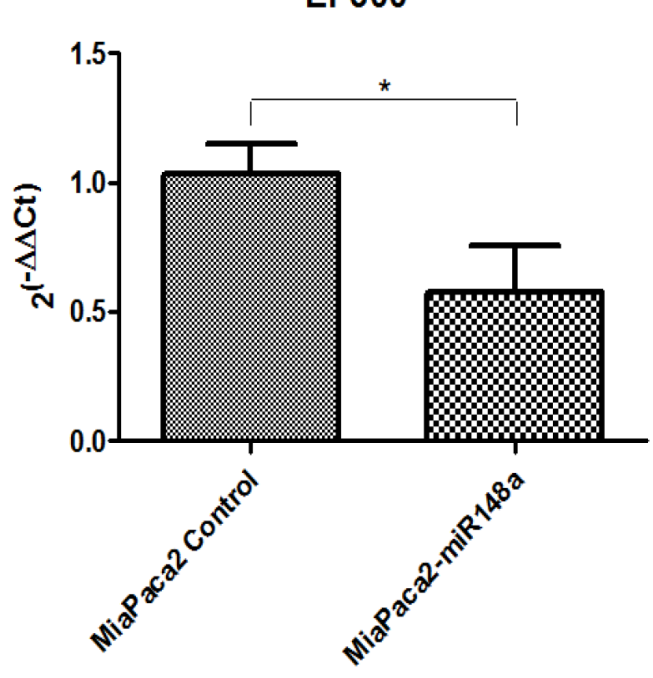

Figure 7: Evaluation of miR-148a targets in a pancreatic cancer cellular model overexpressing miR-148a. (A) qRT-PCR basal expression of miR-148a in the MiaPaca-2 overexpressing miR-148a clone (MiaPaca-2 miR-148a) and MiaPaca-2 scrambled miRNA transfected (MiaPaca-2 Control) (B) qRT-PCR expression of ADAM17 in both MiaPaca-2 Control and MiaPaca-2 miR-148a cells (n=6). (C) qRT-PCR expression of EP300 in both MiaPaca-2 Control and MiaPaca-2 miR-148a cells ( $\mathrm{n}=6$ ). Relative expression of mRNAs was calculated as $2^{(-\Delta \Delta C t)}$. ${ }^{*} \mathrm{P}<0.05,{ }^{* * *} \mathrm{P}<0.001$. 
we used the number of coincidences across databases to prioritize those miRNA-mRNA interactions that would have a more important role in pancreatic carcinogenesis.

Specifically, miRNA-mRNA interactions shown in Table 1 should be those more likely to occur in a pancreatic cancer context, given that they are the most negative correlated that appear simultaneously in the three target prediction databases. Among them, it is noteworthy that there are miRNAs that have been previously described as important in pancreatic cancer for being significantly up-regulated or down-regulated in tumor tissue in comparison to healthy pancreas. For example, miR-106b, miR-107, miR-130a, miR-34 [9], miR-93, miR-155, miR-181a, miR-21, miR-23a, miR320a [8], miR-193b, miR-320b [13] are significantly upregulated and miR-148a [11, 14], miR-330-5p [15], miR373 [16] significantly down-regulated. It is important to highlight the high number of miR-148a interactions that appear among the most significant (12/50), suggesting it may have a central role in pancreatic tumorigenesis. It is likely that miR-148a is involved in more pancreatic cancer pathways than those reported so far for apoptosis

\section{A}

miR-21 expression in PANC-1 KO miR-21

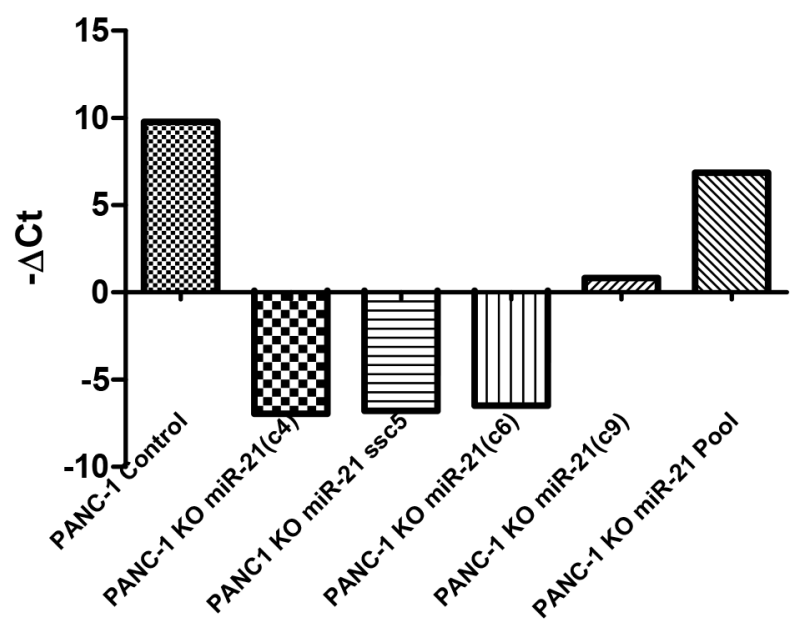

B

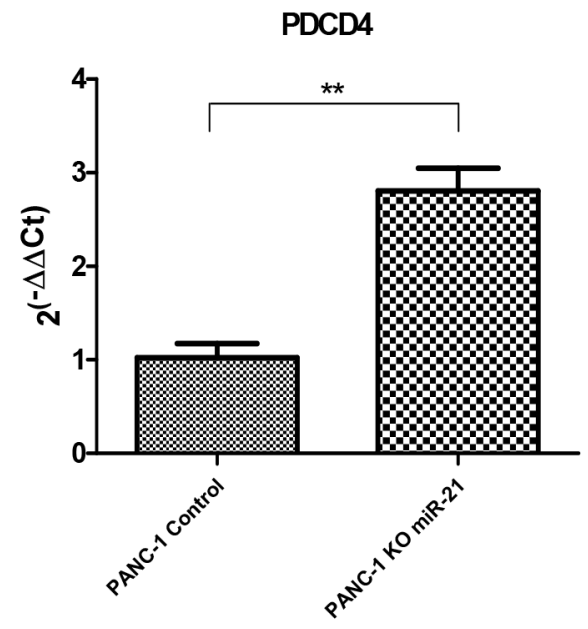

C

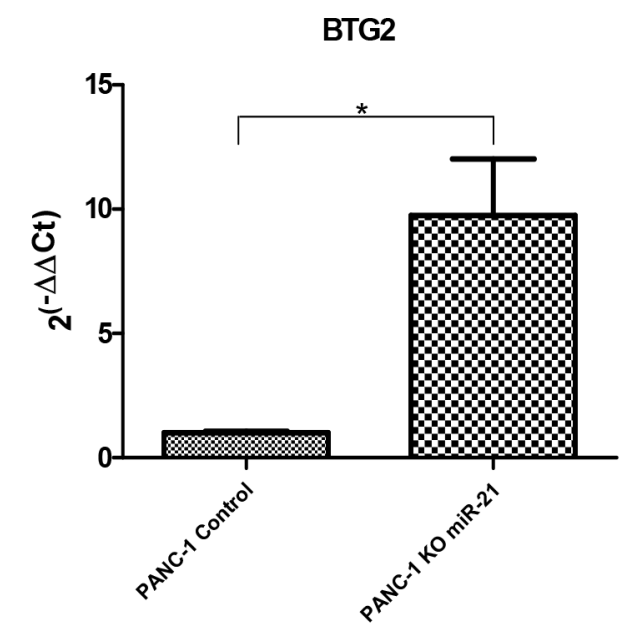

Figure 8: Evaluation of miR-21 targets in a CRISPR/Cas9 generated miR-21 deficient pancreatic cancer cellular model. (A) qRT-PCR expression of miR-21 in the PANC-1 CRISPR/Cas9 generated miR-21 knock-out (KO) clones and PANC-1 Control. (B) qRT-PCR expression of PDCD4 in both PANC-1 Control and PANC-1 KO miR-21 cells (n=3). (C) qRT-PCR expression of BTG2 in both PANC-1 Control and PANC-1 KO miR-21 cells. Relative expression of mRNAs was calculated as $2^{(-\Delta \Delta C t)}$. ${ }^{*} \mathrm{P}<0.05,{ }^{* *} \mathrm{P}<0.01$. 
Table 4: MiR-21 miRComb targets

\begin{tabular}{lccccccc}
\hline miRNA & mRNA & cor & FDR & TargetScan & miRSVR & miRDB & dat.sum \\
\hline miR-21 & PDCD4 & $-0,93$ & 0,010 & 1 & 1 & 1 & 3 \\
miR-21 & PAIP2B & $-0,90$ & 0,012 & 1 & 1 & 0 & 2 \\
miR-21 & SMARCD1 & $-0,88$ & 0,013 & 1 & 1 & 0 & 2 \\
miR-21 & SERP1 & $-0,85$ & 0,015 & 1 & 1 & 0 & 2 \\
miR-21 & B3GAT2 & $-0,84$ & 0,016 & 0 & 1 & 1 & 2 \\
miR-21 & BTG2 & $-0,84$ & 0,016 & 1 & 1 & 0 & 2 \\
miR-21 & BCL7A & $-0,83$ & 0,017 & 1 & 1 & 1 & 3 \\
miR-21 & ALX4 & $-0,83$ & 0,017 & 1 & 0 & 1 & 2 \\
miR-21 & SEC63 & $-0,81$ & 0,019 & 0 & 1 & 1 & 2 \\
miR-21 & RNF182 & $-0,79$ & 0,021 & 0 & 1 & 1 & 2 \\
miR-21 & ARHGAP24 & $-0,79$ & 0,021 & 1 & 1 & 1 & 3 \\
miR-21 & STK40 & $-0,79$ & 0,022 & 1 & 0 & 1 & 2 \\
miR-21 & CNTFR & $-0,78$ & 0,023 & 1 & 1 & 0 & 2 \\
miR-21 & NPAS3 & $-0,77$ & 0,024 & 1 & 1 & 0 & 2 \\
miR-21 & ABAT & $-0,77$ & 0,025 & 0 & 1 & 1 & 2 \\
miR-21 & KLF9 & $-0,76$ & 0,026 & 1 & 1 & 1 & 1 \\
miR-21 & EPM2A & $-0,74$ & 0,028 & 0 & 1 & 1 & 2 \\
miR-21 & ADCY2 & $-0,73$ & 0,030 & 0 & 1 & 1 & 2 \\
miR-21 & PIKFYVE & $-0,70$ & 0,036 & 1 & 1 & 1 & 2 \\
miR-21 & SLC16A10 & $-0,70$ & 0,037 & 1 & 1 & 1 \\
\hline
\end{tabular}

Only significant targets with negative correlation $($ FDR $<0.05)$ and present in 2 or 3 of the databases (TargetScan, miRSVR or miRDB) are shown.

and cell survival $[17,18]$. In that sense, functional enrichment analysis according to miR-148a targets by KEGG, Reactome and GO revealed significant target enrichment in the Notch signaling pathway, among others. Figure 6 shows proteins involved in that Notch pathway highlighting those that appeared as miRComb predicted targets for miR-148a as NUMB, DTX4, DTX3L, PSEN1, APH1A, ADAM17 and EP300. In order to experimentally evaluate these predicted interactions, we analyzed the expression of these targets in a pancreatic cancer cell model overexpressing miR-148a in a stable way. Two components of the Notch signaling pathway, ADAM17 and EP300, could be confirmed as miR-148a targets in that cellular model. In recent years, accumulated evidence has demonstrated that Notch signaling pathway plays critical roles in the development and progression of PDAC [19]. It has been well documented that the Notch signaling pathway is critical for cell proliferation, differentiation, development and homeostasis [20]. Reactivation of Notch signaling is observed in early PDAC pathogenesis and persists throughout the progression of the disease [21-25]. However, no relationships between miR-148a and Notch signaling pathway have been described so far in pancreatic cancer and more studies would be needed to confirm and explore this relationship. Consistently, evidences about miR-148a regulation of Notch pathway members have been recently reported in hepatocellular carcinoma [26].

Concordantly, miR-148a, together with miR-374b, are the miRNAs with more miRComb predicted targets (363 and 381, respectively) as shown in Table 2. MiRNAs appearing in that table probably are those playing more central roles in PDAC because they are the ones with more targets and they would regulate a huge number of mRNAs simultaneously. Interestingly, most of these miRNAs are coincident with those appearing in Table 1 (miR-374b, miR-148a, miR-181a, miR-373, miR-320a, miR-93, miR106b, miR-497, miR-23a, miR-19b, miR-107, miR-15a, miR-330-5p, miR-144), indicating that, apart from being targeting many mRNAs, these miRNAs are participating in the most reliable interactions. Furthermore, as we have 
mentioned above, most of them have already been reported to be significantly deregulated in PDAC. Altogether suggests these miRNAs may constitute central players of pancreatic tumorigenesis and could be new therapeutic target candidates.

Strikingly, the top 10 miRNAs with more targets are able to regulate $41 \%$ of the deregulated mRNAs in PDAC (Table 2). Moreover, $75 \%$ of the mRNAs are regulated by at least one miRNA, and $31.5 \%$ of the mRNAs are regulated by more than 5 miRNAs. We also have to bear in mind that there are mRNAs not regulated by one single miRNA, and that competence and cooperativity between miRNAs have also been described [27, 28]. Altogether, these data confirms that miRNAs are acting as fine-tuning regulators of gene expression in pancreatic cancer as also happens in a wide range of diseases [29-31].

Another important miRNA that seems to play important roles in PDAC is miR-21, as is one of the most deregulated miRNAs in PDAC. MiR-21 is currently one of the best studied miRNAs that plays relevant roles in cancer as it is named as oncomiR-21 [32]. It has also been described to have important roles in pancreatic cancer $[8,33-35]$. In order to experimentally evaluate if some of these predicted targets could act as miR-21 targets in the context of pancreatic cancer, we selected 2 targets (PDCD4, BTG2) from the top miR-21 targets list (Table 4). Both PDCD4 and BTG2 are described to play a tumor suppressor role in several cancers and are downregulated in PDAC [36]. PDCD4 is also a known target of miR-21 in several types of cancer (colon cancer $[37,38]$ or diffuse large B-cell lymphoma [39]), including PDAC [40, 41]. BTG2 has been related to pancreatic cancer [42], and the relation between miR-21 and BTG2 interaction has been observed in other cancers (multiple myeloma [43], liver cancer [44], prostate cancer [45]), but they still have not been directly linked in pancreatic cancer.

In this study we have confirmed the involvement of miR-148a-ADAM17, miR-148a-EP300, miR-21-PDCD4 and miR-21-BTG2 interactions in the pancreatic cancer cell with the help of genetically modified pancreatic cancer cellular models (stable overexpression or CRISPR/ Cas9 knock-out, respectively). However, we cannot affirm that all the interactions proposed here really exist because they should be experimentally validated one by one. Nevertheless, the aim of this study was to unveil a list of high confident miRNA-mRNA interactions for pancreatic cancer that can be the seed for a high number of studies aiming to understand more deeply the molecular pathogenesis of PDAC.

\section{MATERIALS AND METHODS}

\section{Samples}

A set of 12 surgical pancreatic tissue samples (9 PDAC and 3 healthy) from Hospital Clínic of Barcelona
(Barcelona, Spain) patients were included. The same samples were used for both genome-wide miRNA and mRNA profiling. Sample dissection was performed by experienced pathologists who split tissue samples in two different parts: one for gene expression analysis and the other for diagnostic confirmation. Pancreatic tissues were kept on dry ice at all times during handling, flash frozen in liquid nitrogen and stored at $-80^{\circ} \mathrm{C}$ until RNA isolation. Healthy pancreatic samples correspond to the healthy tissue of patients who underwent surgery for other reasons (i.e., ampulloma or neuroendocrine tumours). None of the patients with PDAC had received chemo or radiotherapy before sample collection.

This study was approved by the Institutional Ethics Committee of Hospital Clínic of Barcelona (March 27, 2008) and written informed consent was obtained from all patients in accordance with the Declaration of Helsinki.

Total RNA including miRNA was isolated from frozen macrodissected tissues using the miRNeasy Mini Kit (Qiagen, Valencia, CA, USA), according to the manufacturer protocol. RNA concentrations and purity were evaluated using NanoDrop 1000 Spectrophotometer (Wilmington, DE, USA) and RNA quality was determined by Bioanalyzer 2100 (Agilent, CA, USA).

\section{Data obtention and processing}

Genome-wide miRNA profiling was done by next generation sequencing (NGS) technology on a Genome Analyzer IIx (Illumina, CA, USA) as described in our previous study [8]. MiRNA counts were found according to Mirdeep2 procedure [46]. Reads were aligned to Human Reference Genome GRCh37, and matched to miRBase v.17 in order to find the count miRNAs [47]. Expression was detected for 1733 miRNAs. For this analysis, normalized counts by DESeq [48] were log2scaled in order to apply the LIMMA-trend procedure [49, $50]$ and allow for linear models, as suggested by Law (logcpm values) $[49,50]$.

Matched genome-wide mRNA profiling was analyzed by microarray technology with Human Genome U219 Gene Expression Arrays (Affymetrix, Santa Clara, CA, USA) and normalized according to LIMMA procedure [49].

\section{MiRNA-mRNA interactions}

MiRNA-mRNA correlations were computed using miRComb package [10]. Briefly, this package selects differentially expressed miRNAs and mRNAs from the same sample, computes miRNA-mRNA correlations and, then, matches them with pre-existing target prediction databases. The final selected miRNA-mRNA interactions are those that their expression correlates in a negative and significant manner, and appear as predicted in at least one of the following databases (TargetScan [51] http://targetscan. 
org, miRDB [52] http://mirdb.org/miRDB and miRSVR [53] http://www.microrna.org/microrna/home.do).

KEGG, GO and Reactome enrichment analysis were applied with miRComb R package (which implements the hypergeometric test from GOstats R package). MiR-148a miRComb predicted targets $($ FDR $<0.05)$ detected in at least one database were used. Supplementary Table 3 shows all the results obtained.

\section{Cell culture}

Human pancreatic cancer cell lines PANC-1 and MiaPaca-2 were obtained from European Collection of Cell Cultures (ECACC, Wiltshire, UK) and cultured in Dulbecco's modified Eagle's medium (GIBCO, Thermo Fisher Scientific, Waltham, MA, USA) supplemented with $10 \%$ fetal bovine serum (GIBCO, Thermo Fisher Scientific) and $1 \%$ penicillin/streptomycin (GIBCO, Thermo Fisher Scientific). Cells were incubated at $37^{\circ} \mathrm{C}$ and 5\% $\mathrm{CO} 2$ in a humidified chamber.

\section{MiR-148a overexpression in MiaPaca-2 cells}

MiaPaca-2 cells stably overexpressing miR-148a (MiaPaca-2 miR-148a) and MiaPaca-2 scrambled miRNA transfected (MiaPaca-2 Control) were obtained by us as previously described [11].

\section{CRISPR/Cas9 targeting of miR-21 in PANC-1 cells}

\section{gRNA design}

The gRNA of miR-21 was designed using the "CRISPR design tool" from Feng Zhang Lab (http://crispr. mit.edu/). We chose a PAM sequence in the pre-miR-21 region and selected a 20-bp sequence upstream as the targeting sequence (5'-TCATGGCAACACCAGTCGAT-3'). Oligonucleotides of the indicated sequence were purchased from IDT (Leuven, BE), annealed and cloned into the plentiCRISPRv2 vector following Lentiviral CRISPR Tool box instructions from Zhang Lab deposited to Addgene.

\section{Verification of gRNA-mediated genome cleavage}

HEK293T cells were transfected with the plentiCRISPRv2 containing miR-21 gRNA by CalPhos mammalian transfection kit (Clontech, Takara Bio Company Inc., Mountain View, CA, USA). Cells were treated with $4 \mu \mathrm{g} / \mathrm{ml}$ puromycin for one week. Next, genomic DNA from transfected and wild-type cells was isolated and submitted to PCR amplification of a $555 \mathrm{bp}$ fragment that encompasses miR-21 region using the following primers: Fwd: 5'- CCACACTCTGTCGTATCTGTG-3' Rev: 5'AAGTGCCACCAGACAGAAGG-3'. PCR fragments were subjected to SURVEYOR nuclease assay (Transgenomic) and resolved on $1.5 \%$ agarose gel. Mutations were confirmed by DNA sequencing.

\section{Generation of miR-21-deleted PANC-1 cells}

Lentiviral particles were generated by transfection of vectors plentiCRISPRv2miR-21gRNA or plentiCRISPRv2-Control (for control cells), pVSV-G and pCMV $\triangle 8.91$ into HEK293T by CalPhos mammalian transfection kit. At $48 \mathrm{~h}$ the viral supernatants were collected, filtered and added to PANC-1 cells. Three days after transduction, cells were selected in $8 \mu \mathrm{g} / \mathrm{ml}$ puromycin for one week. Next, limiting dilution was carried out to generate individual clones from PANC-1 infected with miR-21gRNA cells and three weeks later several clones were analyzed for DNA mutation and miR21 expression.

\section{RNA extraction and target expression analysis by qRT-PCR}

Total RNA was isolated from cell cultures using the miRNeasy Mini Kit (Qiagen, Valencia, CA, USA), according to the manufacturer protocol. The final elution volume was $30 \mu \mathrm{L}$. RNA concentrations and purity were evaluated using NanoDrop 1000 Spectrophotometer (Wilmington, DE, USA). Gene expression levels of several targets were analyzed by qRT-PCR using TaqMan High Capacity cDNA Reverse Transcription Kit (Applied Biosystems Inc., Foster City, CA, USA). A two-step protocol involves reverse transcription, followed by a real time PCR with TaqMan probes. Briefly, $1 \mu \mathrm{g}$ total RNA was used per reverse transcription reaction performed in final volume of $10 \mu \mathrm{L}(5 \mu \mathrm{L}$ RNA, $0,4 \mu \mathrm{L}$ of $100 \mathrm{mM}$ dNTPs, $0.5 \mu \mathrm{L}$ of Multiscribe Reverse Transcriptase $(50 \mathrm{U} \mu \mathrm{L}-1)$, $1 \mu \mathrm{L}$ of $10 \mathrm{X}$ RT buffer, $0.5 \mu \mathrm{L}$ of RNase inhibitor (20U $\mu \mathrm{L}-1), 1 \mu \mathrm{L} 10 \mathrm{x}$ RT random primers and 1,6 $\mu \mathrm{L}$ Nucleasefree water) and incubated for: 10 minutes, $25^{\circ} \mathrm{C} ; 120$ minutes, $37^{\circ} \mathrm{C} ; 5$ minutes, $85^{\circ} \mathrm{C}$; hold at $4^{\circ} \mathrm{C}$. The $10 \mu \mathrm{L}$ PCR mixture included $4 \mu \mathrm{L}$ cDNA, $6 \mu \mathrm{L}$ of TaqMan $2 \mathrm{X}$ Universal PCR Master Mix with no AmpErase UNG and $0.5 \mu \mathrm{L}$ of TaqMan $20 \mathrm{X}$ MicroRNA Assay. PCR reactions were incubated in a 384-well optical plate and run on the Viia7 Real-Time PCR System (Applied Biosystems Inc.) as follows: $95^{\circ} \mathrm{C}$ for $10 \mathrm{~min}$ and 50 cycles of $95^{\circ} \mathrm{C}$ for $15 \mathrm{sec}$ and $60^{\circ} \mathrm{C}$ for $1 \mathrm{~min}$. All specimens were amplified in triplicates. Amplification data was normalized against Cyclophilin as endogenous control. $\mathrm{Ct}$ values were calculated from automatic threshold. No template controls showed any amplification. Relative expression levels of mRNAs versus control cell lines expressing levels were

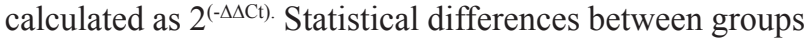
were computed by using T-test.

\section{Abbreviations}

PDAC: pancreatic ductal adenocarcinoma; FDR: false discovery rate; miRNA: microRNA; NES: normalized enrichment score; FC: fold-change; $\mathrm{KO}$ : knock-out. 


\section{Author contributions}

MVC, JJL and MG designed the study; MVC performed experiments and bioinformatic analysis; EVN, GR, CF designed and performed CRISPR-Cas9 experiments; $\mathrm{AC}$ provided samples and supervised the study; MVC, JJL and MG analyzed the data; MVC and MG wrote the manuscript.

\section{ACKNOWLEDGMENTS}

We acknowledge the Genome Analysis Platform of CICbioGUNE (Derio, Spain) for the miR-Sequencing services, the Functional Genomics core facility of IDIBAPS for the microarray services, and the Biobank core facility of the IDIBAPS (Barcelona, Spain).

\section{CONFLICTS OF INTEREST}

There are no conflicts of interest to disclose.

\section{FUNDING}

The present work was supported by grants from Instituto de Salud Carlos III (PI13/02192,), Fundación Científica de la Asociación Española contra el Cáncer (GCB13131592CAST) and Ministerio de Economía y Competitividad (RTC-2015-3850-1, SAF201454453-R and BIO2014-57716-C2-2-R) and co-funded by Fondo Europeo de Desarrollo Nacional (FEDER). Additional support came from Generalitat de Catalunya SGR14/248 and SGR14/135. CIBEREHD and CIBERER are funded by the Instituto de Salud Carlos III. We also acknowledge the support of CERCA Programme/ Generalitat de Catalunya. This work was developed at the Centro Esther Koplowitz, Barcelona, Spain. MVC is funded by Ministerio de Educación Cultura y Deporte (FPU12/05138). GR received an FPI fellowship from Ministerio de Economía y Competitividad.

\section{REFERENCES}

1. American Cancer Society. Cancer Facts \& Figures 2013. [cited 2017 May 11]. Available from https://www.cancer. org/research/cancer-facts-statistics/all-cancer-facts-figures/ cancer-facts-figures-2013.html.

2. Stathis A, Moore MJ. Advanced pancreatic carcinoma: current treatment and future challenges. Nat Rev Clin Oncol. 2010; 7:163-72. https://doi.org/10.1038/ nrclinonc.2009.236.

3. Raimondi S, Maisonneuve P, Lowenfels AB. Epidemiology of pancreatic cancer: an overview. Nat Rev Gastroenterol Hepatol. 2009; 6:699-708. https://doi.org/10.1038/ nrgastro.2009.177.
4. Bartel DP. MicroRNA target recognition and regulatory functions. Cell. 2009; 136:215-33. https://doi.org/10.1016/j. cell.2009.01.002.

5. Esteller M. Non-coding RNAs in human disease. Nat Rev Genet. 2011; 12:861-74. https://doi.org/10.1038/nrg3074.

6. Leva GD, Garofalo M, Croce CM. MicroRNAs in cancer. Annu Rev Pathol. 2014; 9:287-314. https://doi.org/10.1146/ annurev-pathol-012513-104715.

7. Esquela-Kerscher A, Slack FJ. Oncomirs - microRNAs with a role in cancer. Nat Rev Cancer. 2006; 6:259-69. https://doi.org/10.1038/nrc1840.

8. Vila-Navarro E, Vila-Casadesús M, Moreira L, DuranSanchon S, Sinha R, Ginés A, Fernández-Esparrach G, Miquel R, Cuatrecasas M, Castells A, Lozano JJ, Gironella M. MicroRNAs for detection of pancreatic neoplasia: biomarker discovery by next-generation sequencing and validation in 2 independent cohorts. Ann Surg. 2017; 265:1226-34. https://doi.org/10.1097/SLA. OT_037461_3_msOT_037461_3_ms1809.

9. Jamieson NB, Morran DC, Morton JP, Ali A, Dickson EJ, Carter CR, Sansom OJ, Evans TR, McKay CJ, Oien KA. MicroRNA molecular profiles associated with diagnosis, clinicopathologic criteria, and overall survival in patients with resectable pancreatic ductal adenocarcinoma. Clin Cancer Res. 2012; 18:534-45. https://doi.org/10.1158/10780432.CCR-11-0679.

10. Vila-Casadesús M, Gironella M, Lozano JJ. MiRComb: an $\mathrm{R}$ package to analyse miRNA-mRNA interactions. Examples across five digestive cancers. PLoS One. 2016; 11:e0151127. https://doi.org/10.1371/journal. pone. 0151127.

11. Bofill-De Ros X, Gironella M, Fillat C. miR-148a- and miR-216a-regulated oncolytic adenoviruses targeting pancreatic tumors attenuate tissue damage without perturbation of miRNA activity. Mol Ther. 2014; 22:166577. https://doi.org/10.1038/mt.2014.98.

12. Thomson DW, Bracken CP, Goodall GJ. Experimental strategies for microRNA target identification. Nucleic Acids Res. 2011; 39:6845-53. https://doi.org/10.1093/nar/gkr330.

13. Liu R, Chen X, Du Y, Yao W, Shen L, Wang C, Hu Z, Zhuang R, Ning G, Zhang C, Yuan Y, Li Z, Zen K, et al. Serum microRNA expression profile as a biomarker in the diagnosis and prognosis of pancreatic cancer. Clin Chem. 2012; 58:610-8. https://doi.org/10.1373/ clinchem.2011.172767.

14. Hanoun N, Delpu Y, Suriawinata AA, Bournet B, Bureau C, Selves J, Tsongalis GJ, Dufresne M, Buscail L, Cordelier P, Torrisani J. The silencing of microRNA 148a production by DNA hypermethylation is an early event in pancreatic carcinogenesis. Clin Chem. 2010; 56:1107-18. https://doi. org/10.1373/clinchem.2010.144709.

15. Tréhoux S, Lahdaoui F, Delpu Y, Renaud F, Leteurtre E, Torrisani J, Jonckheere N, Van Seuningen I. Micro-RNAs miR-29a and miR-330-5p function as tumor suppressors 
by targeting the MUC1 mucin in pancreatic cancer cells. Biochim Biophys Acta. 2015; 1853:2392-403. https://doi. org/10.1016/j.bbamcr.2015.05.033.

16. Nakata K, Ohuchida K, Mizumoto K, Aishima S, Oda Y, Nagai E, Tanaka M. Micro RNA-373 is down-regulated in pancreatic cancer and inhibits cancer cell invasion. Ann Surg Oncol. 2014; 21:S564-74. https://doi.org/10.1245/ s10434-014-3676-8.

17. Liffers ST, Munding JB, Vogt M, Kuhlmann JD, Verdoodt B, Nambiar S, Maghnouj A, Mirmohammadsadegh A, Hahn SA, Tannapfel A. MicroRNA-148a is down-regulated in human pancreatic ductal adenocarcinomas and regulates cell survival by targeting CDC25B. Lab Invest. 2011; 91:1472-9. https://doi.org/10.1038/labinvest.2011.99.

18. Zhang R, Li M, Zang W, Chen X, Wang Y, Li P, Du Y, Zhao $\mathrm{G}, \mathrm{Li}$ L. MiR-148a regulates the growth and apoptosis in pancreatic cancer by targeting CCKBR and Bcl-2. Tumour Biol. 2014; 35:837-44. https://doi.org/10.1007/ s13277-013-1115-2.

19. Gao J, Long B, Wang Z. Role of Notch signaling pathway in pancreatic cancer. Am J Cancer Res. 2017; 7:173-86.

20. Ranganathan P, Weaver KL, Capobianco AJ. Notch signalling in solid tumours: a little bit of everything but not all the time. Nat Rev Cancer. 2011; 11:338-51. https://doi. org/10.1038/nrc3035.

21. Tremblay I, Paré E, Arsenault D, Douziech M, Boucher MJ. The MEK/ERK pathway promotes NOTCH signalling in pancreatic cancer cells. PLoS One. 2013; 8:e85502. https:// doi.org/10.1371/journal.pone.0085502.

22. Hingorani SR, Petricoin EF, Maitra A, Rajapakse V, King C, Jacobetz MA, Ross S, Conrads TP, Veenstra TD, Hitt BA, Kawaguchi Y, Johann D, Liotta LA, et al. Preinvasive and invasive ductal pancreatic cancer and its early detection in the mouse. Cancer Cell. 2003; 4:437-50. https://doi. org/10.1016/S1535-6108(03)00309-X.

23. Miyamoto Y, Maitra A, Ghosh B, Zechner U, Argani P, Iacobuzio-Donahue CA, Sriuranpong V, Iso T, Meszoely IM, Wolfe MS, Hruban RH, Ball DW, Schmid RM, et al. Notch mediates TGF\$ $\backslash$ alpha\$-induced changes in epithelial differentiation during pancreatic tumorigenesis. Cancer Cell. 2003; 3:565-76. https://doi.org/10.1016/ S1535-6108(03)00140-5.

24. Pasca di Magliano M, Sekine S, Ermilov A, Ferris J, Dlugosz AA, Hebrok M. Hedgehog/Ras interactions regulate early stages of pancreatic cancer. Genes Dev. 2006; 20:3161-73. https://doi.org/10.1101/gad.1470806.

25. Stanger BZ, Stiles B, Lauwers GY, Bardeesy N, Mendoza M, Wang Y, Greenwood A, Cheng K, McLaughlin M, Brown D, DePinho RA, Wu H, Melton DA, et al. Pten constrains centroacinar cell expansion and malignant transformation in the pancreas. Cancer Cell. 2005; 8:18595. https://doi.org/10.1016/j.ccr.2005.07.015.
26. Jung KH, Zhang J, Zhou C, Shen H, Gagea M, RodriguezAguayo C, Lopez-Berestein G, Sood AK, Beretta L. Differentiation therapy for hepatocellular carcinoma: multifaceted effects of miR-148a on tumor growth and phenotype and liver fibrosis. Hepatology. 2016; 63:864-79. https://doi.org/10.1002/hep.28367.

27. Nielsen CB, Shomron N, Sandberg R, Hornstein E, Kitzman $\mathrm{J}$, Burge CB. Determinants of targeting by endogenous and exogenous microRNAs and siRNAs. RNA. 2007; 13:1894910. https://doi.org/10.1261/rna.768207.

28. Grimson A, Farh KK, Johnston WK, Garrett-Engele P, Lim LP, Bartel DP. MicroRNA targeting specificity in mammals: determinants beyond seed pairing. Mol Cell. 2007; 27:91105. https://doi.org/10.1016/j.molcel.2007.06.017.

29. Sevignani C, Calin GA, Siracusa LD, Croce CM. Mammalian microRNAs: a small world for fine-tuning gene expression. Mamm Genome. 2006; 17:189-202. https://doi. org/10.1007/s00335-005-0066-3.

30. Wu CT, Chiou CY, Chiu HC, Yang UC. Fine-tuning of microRNA-mediated repression of mRNA by splicingregulated and highly repressive microRNA recognition element. BMC Genomics. 2013; 14:438. https://doi. org/10.1186/1471-2164-14-438.

31. Lai X, Wolkenhauer O, Vera J. Understanding microRNAmediated gene regulatory networks through mathematical modelling. Nucleic Acids Res. 2016; 44:6019-35. https:// doi.org/10.1093/nar/gkw550.

32. Pfeffer SR, Yang CH, Pfeffer LM. The role of miR-21 in cancer. Drug Dev Res. 2015; 76:270-7. https://doi. org/10.1002/ddr.21257.

33. Giovannetti E, Funel N, Peters GJ, Chiaro MD, Erozenci LA, Vasile E, Leon LG, Pollina LE, Groen A, Falcone A, Danesi R, Campani D, Verheul HM, et al. MicroRNA-21 in pancreatic cancer: correlation with clinical outcome and pharmacologic aspects underlying its role in the modulation of gemcitabine activity. Cancer Res. 2010; 70:4528-38. https://doi.org/10.1158/0008-5472.CAN-09-4467.

34. Dillhoff M, Liu J, Frankel W, Croce C, Bloomston M. MicroRNA-21 is overexpressed in pancreatic cancer and a potential predictor of survival. J Gastrointest Surg. 2008; 12:2171-6. https://doi.org/10.1007/s11605-008-0584-x.

35. Moriyama $\mathrm{T}$, Ohuchida $\mathrm{K}$, Mizumoto $\mathrm{K}, \mathrm{Yu} \mathrm{J}$, Sato N, Nabae T, Takahata S, Toma H, Nagai E, Tanaka M. MicroRNA-21 modulates biological functions of pancreatic cancer cells including their proliferation, invasion, and chemoresistance. Mol Cancer Ther. 2009; 8:1067-74. https://doi.org/10.1158/1535-7163.MCT-08-0592.

36. Tan ZJ, Hu XG, Cao GS, Tang Y. Analysis of gene expression profile of pancreatic carcinoma using cDNA microarray. World J Gastroenterol. 2003; 9:818-23. https:// doi.org/10.3748/wjg.v9.i4.818.

37. Asangani IA, Rasheed SA, Nikolova DA, Leupold JH, Colburn NH, Post S, Allgayer H. MicroRNA-21 (miR-21) 
post-transcriptionally downregulates tumor suppressor Pdcd 4 and stimulates invasion, intravasation and metastasis in colorectal cancer. Oncogene. 2008; 27:2128-36. https:// doi.org/10.1038/sj.onc. 1210856 .

38. Peacock O, Lee AC, Cameron F, Tarbox R, VafadarIsfahani N, Tufarelli C, Lund JN. Inflammation and miR-21 pathways functionally interact to downregulate PDCD4 in colorectal cancer. PLoS One. 2014; 9:e110267. https://doi. org/10.1371/journal.pone.0110267.

39. Gu L, Song G, Chen L, Nie Z, He B, Pan Y, Xu Y, Li R, Gao T, Cho WC, Wang S. Inhibition of miR-21 induces biological and behavioral alterations in diffuse large B-cell lymphoma. Acta Haematol. 2013; 130:87-94. https://doi. org/10.1159/000346441.

40. Nagao Y, Hisaoka M, Matsuyama A, Kanemitsu S, Hamada T, Fukuyama T, Nakano R, Uchiyama A, Kawamoto M, Yamaguchi K, Hashimoto H. Association of microRNA-21 expression with its targets, PDCD4 and TIMP3, in pancreatic ductal adenocarcinoma. Mod Pathol. 2012; 25:112-21. https://doi.org/10.1038/modpathol.2011.142.

41. Roldo C, Missiaglia E, Hagan JP, Falconi M, Capelli P, Bersani S, Calin GA, Volinia S, Liu CG, Scarpa A, Croce CM. MicroRNA expression abnormalities in pancreatic endocrine and acinar tumors are associated with distinctive pathologic features and clinical behavior. J Clin Oncol. 2006; 24:4677-84. https://doi.org/10.1200/ JCO.2005.05.5194.

42. Jin $\mathrm{G}, \mathrm{Hu} \mathrm{XG}$, Ying $\mathrm{K}$, Tang Y, Liu R, Zhang YJ, Jing ZP, Xie Y, Mao YM. Discovery and analysis of pancreatic adenocarcinoma genes using cDNA microarrays. World J Gastroenterol. 2005; 11:6543-8.

43. Leone E, Morelli E, Di Martino MT, Amodio N, Foresta U, Gullà A, Rossi M, Neri A, Giordano A, Munshi NC, Anderson KC, Tagliaferri P, Tassone P. Targeting miR21 inhibits in vitro and in vivo multiple myeloma cell growth. Clin Cancer Res. 2013; 19:2096-106. https://doi. org/10.1158/1078-0432.CCR-12-3325.

44. Mao B, Xiao H, Zhang Z, Wang D, Wang G. MicroRNA-21 regulates the expression of BTG2 in HepG2 liver cancer cells. Mol Med Rep. 2015; 12:4917-24. https://doi. org/10.3892/mmr.2015.4051.

45. Coppola V, Musumeci M, Patrizii M, Cannistraci A, Addario A, Maugeri-Saccà M, Biffoni M, Francescangeli F,
Cordenonsi M, Piccolo S, Memeo L, Pagliuca A, Muto G, et al. Btg2 loss and miR-21 upregulation contribute to prostate cell transformation by inducing luminal markers expression and epithelial-mesenchymal transition. Oncogene. 2013; 32:1843-53. https://doi.org/10.1038/onc.2012.194.

46. Friedländer MR, Mackowiak SD, Li N, Chen W, Rajewsky N. miRDeep2 accurately identifies known and hundreds of novel microRNA genes in seven animal clades. Nucleic Acids Res. 2012; 40:37-52. https://oi.org/10.1093/nar/ gkr688.

47. Griffiths-Jones S, Saini HK, van Dongen S, Enright AJ. miRBase: tools for microRNA genomics. Nucleic Acids Res. 2008; 36:D154-8. https://doi.org/10.1093/nar/gkm952.

48. Anders S, Huber W. Differential expression analysis for sequence count data. Genome Biol. 2010; 11:R106. https:// doi.org/10.1186/gb-2010-11-10-r106.

49. Smyth GK. limma: Linear Models for Microarray Data. In: Gentleman R, Carey VJ, Huber W, Irizarry RA, Dudoit $\mathrm{S}$, editors. Bioinformatics and Computational Biology Solutions Using R and Bioconductor. Springer, New York; 2005. [cited 2014 Oct 24]. p. 397-420. Available from http:// link.springer.com/chapter/10.1007/0-387-29362-0_23.

50. Law CW, Chen Y, Shi W, Smyth GK. voom: precision weights unlock linear model analysis tools for RNA-seq read counts. Genome Biol. 2014; 15:R29. https://doi. org/10.1186/gb-2014-15-2-r29.

51. Lewis BP, Burge CB, Bartel DP. Conserved seed pairing, often flanked by adenosines, indicates that thousands of human genes are microRNA targets. Cell. 2005; 120:15-20. https://doi.org/10.1016/j.cell.2004.12.035.

52. Wong N, Wang X. miRDB: an online resource for microRNA target prediction and functional annotations. Nucleic Acids Res. 2015; 43:D146-52. https:/doi. org/10.1093/nar/gku1104.

53. Betel D, Koppal A, Agius P, Sander C, Leslie C. Comprehensive modeling of microRNA targets predicts functional non-conserved and non-canonical sites. Genome Biol. 2010; 11:R90. https://doi.org/10.1186/ gb-2010-11-8-r90. 